\title{
Poisson structures on the Poincaré group
}

\author{
S. Zakrzewski \\ Department of Mathematical Methods in Physics, University of Warsaw \\ Hoża 74, 00-682 Warsaw, Poland
}

\begin{abstract}
An introduction to inhomogeneous Poisson groups is given. Poisson inhomogeneous $O(p, q)$ are shown to be coboundary, the generalized classical Yang-Baxter equation having only one-dimensional right hand side. Normal forms of the classical $r$-matrices for the Poincaré group (inhomogeneous $O(1,3)$ ) are calculated.
\end{abstract}

\section{Introduction}

In this paper we give the proofs of facts announced in our previous article [1], in which we have presented a list of 23 normal forms of classical $r$-matrices on the Poincaré group.

It is remarkable that the classification of Poisson Poincaré groups turns out to be completely analogous to the classification of quantum Poincaré groups given in [2]. Recall also that the main motivation of these investigations is the potential possibility of deforming the relativistic symmetry.

The paper is organized as follows. In Sect. 1 we recall basic definitions and facts concerning Lie bialgebras, especially in the context of semi-direct product Lie algebras. In Sect. 2 we prove that inhomogeneous $o(p, q)$ Lie algebras have the following interesting features of simple Lie algebras: all Lie bialgebra structures are coboundary (all Poisson Lie structures are of the $r$-matrix type) and the subspace of invariants in the third antisymmetric tensor power of the Lie algebra (the right hand side of the generalized classical Yang-Baxter equation) is only one-dimensional. We formulate a set of equations determining the classical $r$-matrix and present some solutions.

In Sect. 3 we restrict ourselves to the case of the Poincaré Lie algebra (inhomogeneous $o(1,3))$ and present the main result: a table of solutions. Sections 4 and 5 are devoted to the proofs.

All vector spaces and Lie algebras considered in this paper are real and finite-dimensional.

\section{Preliminaries}

\section{$1.1 \quad$ Modules}

Let $\mathfrak{g}$ be a Lie algebra and let $E$ be a vector space. Recall that $E$ is a $\mathfrak{g}$-module if a bilinear map

$$
\mathfrak{g} \times E \ni(X, u) \mapsto X u \in E
$$


is given, such that $[X, Y] u=X(Y u)-Y(X u)$ for $X, Y \in \mathfrak{g}, u \in E$. We denote by $E_{\mathfrak{g}}$ the subspace of invariant elements:

$$
E_{\mathfrak{g}}:=\{u \in E: X u=0 \text { for } X \in \mathfrak{g}\} .
$$

A morphism from a $\mathfrak{g}$-module $E_{1}$ to a $\mathfrak{g}$-module $E_{2}$ is a linear map $T: E_{1} \rightarrow E_{2}$ such that $T(X u)=X T(u)$ for $X \in \mathfrak{g}, u \in E_{1}$. The linear space of morphisms from $E_{1}$ to $E_{2}$ is denoted by $\operatorname{Mor}_{\mathfrak{g}}\left(E_{1}, E_{2}\right)$. We have also the well known alternative terminology:

$$
\begin{aligned}
\mathfrak{g} \text {-modules } & =\text { representations of } \mathfrak{g} \\
\text { morphisms of } \mathfrak{g} \text {-modules } & =\text { intertwiners. }
\end{aligned}
$$

The tensor product of modules (representations) is naturally defined. An important example of the $\mathfrak{g}$-module is $\mathfrak{g}$ itself with the adjoint action: $X Y:=[X, Y]$. For the purpose of this paper, the most important $\mathfrak{g}$-module will be $\stackrel{2}{\wedge} \mathfrak{g}$.

\subsection{Cocycles and coboundaries}

Let $E$ be a $\mathfrak{g}$-module. Linear map $f$ from $\mathfrak{g}$ to $E$ is a cocycle (on $\mathfrak{g}$ with values in $E$ ) if

$$
f([X, Y])=X f(Y)-Y f(X)
$$

for $X, Y \in \mathfrak{g}$. The space of cocycles on $\mathfrak{g}$ with values in $E$ is denoted by $Z(\mathfrak{g}, E)$. If $r \in E$, then the linear map

$$
\mathfrak{g} \ni X \mapsto(\partial r)(X):=X r \in E
$$

is said to be the coboundary of $r$. Coboundaries of elements of $E$ form a subspace in $Z(\mathfrak{g}, E)$ which is denoted by $B(\mathfrak{g}, E)$. We set

$$
H(\mathfrak{g}, E):=Z(\mathfrak{g}, E) / B(\mathfrak{g}, E) .
$$

Note that $H(\mathfrak{g}, E)=\{0\}$ if and only if each cocycle is a coboundary. The well known Whitehead's lemma says that for semisimple $\mathfrak{g}$ and arbitrary $\mathfrak{g}$-module $E$ we have $H(\mathfrak{g}, E)=$ $\{0\}$.

In order to approach the case of semi-direct product Lie algebras, let us note the following useful (very simple) facts.

1. The restriction of a cocycle to a Lie subalgebra is a cocycle (on this subalgebra).

2. If the restriction of a cocycle $\delta \in Z(\mathfrak{g}, E)$ to a Lie subalgebra $\mathfrak{h}$ is a coboundary, i.e. there exists $r \in E$ such that $\delta(X)=X r$ for $X \in \mathfrak{h}$, then

$$
\delta_{0}:=\delta-\partial r \in Z(\mathfrak{g}, E) \quad \text { satisfies }\left.\quad \delta_{0}\right|_{\mathfrak{h}}=0
$$

3. Let $\mathfrak{g}=\mathfrak{n} \rtimes \mathfrak{h}$ (semidirect product; $\mathfrak{n}$ is the ideal) and let $\delta_{0}: \mathfrak{g} \rightarrow E$ be a linear map. Then

$$
\delta_{0} \in Z(\mathfrak{g}, E),\left.\quad \delta_{0}\right|_{\mathfrak{h}}=\left.0 \Longleftrightarrow \delta_{0}\right|_{\mathfrak{n}} \in Z(\mathfrak{n}, E) \cap \operatorname{Mor}_{\mathfrak{h}}(\mathfrak{n}, E)
$$


4. For $\mathfrak{g}=\mathfrak{n} \rtimes \mathfrak{h}$ and $E:=\stackrel{2}{\wedge} \mathfrak{g}$, we have

$$
\begin{gathered}
E=\bigwedge^{2} \mathfrak{n} \oplus(\mathfrak{n} \otimes \mathfrak{h}) \oplus \bigwedge^{2} \mathfrak{h} \quad(\mathfrak{h} \text {-invariant decomposition }) \\
\operatorname{Mor}_{\mathfrak{h}}(\mathfrak{n}, E)=\operatorname{Mor}_{\mathfrak{h}}\left(\mathfrak{n}, \bigwedge^{2} \mathfrak{n}\right) \oplus \operatorname{Mor}_{\mathfrak{h}}(\mathfrak{n}, \mathfrak{n} \otimes \mathfrak{h}) \oplus \operatorname{Mor}_{\mathfrak{h}}\left(\mathfrak{n}, \bigwedge^{2} \mathfrak{h}\right)
\end{gathered}
$$

Example 1.1 If $\mathfrak{h}$ is semi-simple and $\mathfrak{g}:=\mathbb{R} \oplus \mathfrak{h}$, then $H(\mathfrak{g}, \stackrel{2}{\wedge} \mathfrak{g})=\{0\}$

Proof: If $\delta \in Z(\mathfrak{g}, \stackrel{2}{\wedge} \mathfrak{g})$ then $\left.\delta\right|_{\mathfrak{h}} \in B(\mathfrak{h}, \stackrel{2}{\wedge} \mathfrak{g})$ (Whitehead's lemma), i.e. there exists $r \in \bigwedge^{2} \mathfrak{g}$ such that $\delta(X)=X r$ for $X \in \mathfrak{h}$. Setting $\delta_{0}:=\delta-\partial r$ and using points 2 and 3 above we see that $\delta_{0} \in \operatorname{Mor}_{\mathfrak{h}}(\mathbb{R}, \stackrel{2}{\wedge} \mathfrak{g})$. Note that

$$
\mathfrak{h}_{\mathfrak{h}}=\{0\}, \quad\left(\bigwedge^{2} \mathfrak{h}\right)_{\mathfrak{h}}=\{0\}
$$

(for the last equality, see e.g. [3], Thm.I, p. 189). It follows that

$$
\operatorname{Mor}_{\mathfrak{h}}\left(\mathbb{R}, \bigwedge^{2} \mathfrak{g}\right)=\operatorname{Mor}_{\mathfrak{h}}(\mathbb{R}, \mathfrak{h}) \oplus \operatorname{Mor}_{\mathfrak{h}}\left(\mathbb{R}, \bigwedge^{2} \mathfrak{h}\right)=\mathfrak{h}_{\mathfrak{h}} \oplus\left(\bigwedge^{2} \mathfrak{h}\right)_{\mathfrak{h}}=\{0\}
$$

Q.E.D.

Analogous fact holds of course for complex Lie algebras (with $\mathbb{R}$ replaced by $\mathbb{C}$ ).

\subsection{Lie bialgebras}

Recall [4 [5] that a Lie bialgebra is a Lie algebra $\mathfrak{g}$ together with a cocycle $\delta: \mathfrak{g} \rightarrow \stackrel{2}{\wedge} \mathfrak{g}$ such that the dual map $\delta^{*}: \bigwedge^{2} \mathfrak{g}^{*} \rightarrow \mathfrak{g}^{*}$ is a Lie bracket on $\mathfrak{g}^{*}$ (the dual of $\mathfrak{g}$ ). There is a $1-1$ correspondence between Lie bialgebras and connected, simply connected Poisson Lie groups 4, 5, 6, 7].

A Lie bialgebra $(\mathfrak{g}, \delta)$ is said to be coboundary if $\delta$ is a coboundary: $\delta=\partial r, r \in \bigwedge^{2} \mathfrak{g}$. Of course, Lie bialgebras $(\mathfrak{g}, \delta)$ with $\mathfrak{g}$ semisimple are always coboundary. A non-semisimple Lie algebra with the same property is provided by Example 1.1 and the following special case of it.

Example 1.2 Any Lie bialgebra structure on $\mathfrak{g}=g l(n)$ is coboundary.

If $\delta=\partial r$ then $\delta^{*}$ is a Lie bracket if and only if

$$
[r, r] \in\left(\bigwedge^{3} \mathfrak{g}\right)_{\mathfrak{g}}
$$

(the bracket used here is the Schouten bracket). Condition (7) is called generalized classical Yang-Baxter equation and $r$ is said to be a classical $r$-matrix. The Lie bracket defined by $\partial r$ on $\mathfrak{g}^{*}$ equals

$$
[\alpha, \beta]_{r}=r(\alpha) \beta-r(\beta) \alpha, \quad \alpha, \beta \in \mathfrak{g}^{*}
$$


where $r(\cdot): \mathfrak{g}^{*} \rightarrow \mathfrak{g}$ is the contraction with $r$ from the left:

$$
r(\alpha):=\alpha\lrcorner r, \quad\langle r(\alpha), \beta\rangle=\langle r, \alpha \otimes \beta\rangle, \quad \alpha, \beta \in \mathfrak{g}^{*} .
$$

If $r$ is triangular, i.e. $[r, r]=0$, then $r(\cdot)$ is a Lie algebra homomorphism:

$$
r\left([\alpha, \beta]_{r}\right)=[r(\alpha), r(\beta)] .
$$

This is a consequence of the following useful formula:

$$
\frac{1}{2}\langle[r, r], \alpha \wedge \beta \wedge \gamma\rangle=\left\langle[r(\alpha), r(\beta)]-r\left([\alpha, \beta]_{r}\right), \gamma\right\rangle, \quad \alpha, \beta \gamma \in \mathfrak{g}^{*} .
$$

\section{Inhomogeneous $o(p, q)$ algebras}

We consider a $(p+q)$-dimensional real vector space $V \cong \mathbb{R}^{p+q}$, equipped with a scalar product $g$ of signature $(p, q)$. Let $\mathfrak{h}:=o(p, q)$ denote the Lie algebra of the group $O(p, q)$ of endomorphisms of $V$ preserving $g$. Let $\mathfrak{g}:=V \rtimes \mathfrak{h}$ be the corresponding 'inhomogeneous' Lie algebra.

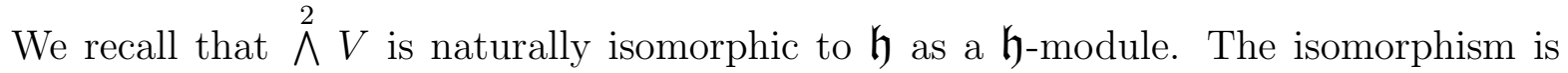
given by $\Omega:=$ id $\otimes g$ (here $g$ is interpreted as a map from $V$ to $V^{*}$ ). For $x, y \in V$ we set

$$
\Omega_{x, y}:=\Omega(x \wedge y)=x \otimes g(y)-y \otimes g(x) \in \mathfrak{h} \subset \text { End } V .
$$

When working with a basis $e_{1}, \ldots, e_{p+q}$ of $V$, we shall use also the following notation

$$
\Omega_{j, k}:=\Omega_{e_{j}, e_{k}}=\left(g_{k l} e_{j}-g_{j l} e_{k}\right) \otimes e^{l}, \quad j, k=1, \ldots, p+q
$$

(summation convention), where $e^{1}, \ldots, e^{p+q}$ is the dual basis and $g_{j k}:=g\left(e_{j}, e_{k}\right)$.

Theorem 2.1 For $\operatorname{dim} V>2$ we have

$$
\begin{aligned}
H\left(\mathfrak{g}, \bigwedge^{2} \mathfrak{g}\right) & =\{0\} & \text { (i.e. any cocycle } \delta: \mathfrak{g} & \rightarrow \stackrel{\Upsilon}{\Lambda}^{2} \mathfrak{g} \text { is a coboundary) } \\
\left(\bigwedge^{2} \mathfrak{g}\right)_{\mathfrak{g}} & =\{0\} & & (r \mapsto \partial r \text { is injective }) .
\end{aligned}
$$

Proof: Note that $\mathfrak{h}=o(p, q)$ is semisimple for $p+q>2$ (it is even simple, except the case $o(4,0)=o(3,0) \oplus o(3,0)$ and $o(2,2)=o(2,1) \oplus o(2,1))$. We first prove (15). Indeed, using (3) with $\mathfrak{n}=V$ and

$$
\left(\bigwedge^{2} V\right)_{\mathfrak{h}} \cong(\mathfrak{h})_{\mathfrak{h}}=\{0\}, \quad\left(\bigwedge^{2} \mathfrak{h}\right)_{\mathfrak{h}}=\{0\}
$$

(as in (5)), we have

$$
\left(\bigwedge^{2} \mathfrak{g}\right)_{\mathfrak{h}}=(V \otimes \mathfrak{h})_{\mathfrak{h}} \cong \operatorname{Mor}_{\mathfrak{h}}(V, \mathfrak{h})
$$

The latter space is $\{0\}$ if $\operatorname{dim} V>3$ (because $V$ and $\mathfrak{h}$ are irreducible $\mathfrak{h}$-modules of different dimension; only $\mathfrak{h}=o(4,0), \mathfrak{h}=o(2,2)$ are reducible, but in this case the irreducible $\mathfrak{h}$-submodules are of dimension 3). If $\operatorname{dim} V=3$, we have $\mathfrak{h} \cong V$ and

$$
\operatorname{Mor}_{\mathfrak{h}}(V, \mathfrak{h})=\operatorname{Mor}_{\mathfrak{h}}(V, V) \cong \mathbb{R} \text {. }
$$


It is easy to check that in this case the non-zero $\mathfrak{h}$-invariant element

$$
s:=\varepsilon^{j k l} e_{j} \otimes \Omega_{k l}
$$

of $V \otimes \mathfrak{h} \subset \wedge^{2} \mathfrak{g}$ is not $V$-invariant (here $\varepsilon^{j k l}$ is the usual antisymmetric symbol). Concluding,

$$
\left(\bigwedge^{2} \mathfrak{g}\right)_{\mathfrak{g}}=\{0\} .
$$

To prove (14), it is sufficient (in view of (1),(2) and semisimplicity of $\mathfrak{h}$ ) to show that if

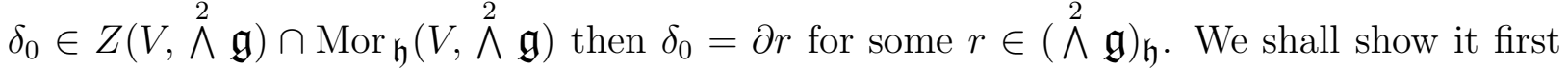
for $p+q>3$ (we shall actually show that $\delta_{0}=0$ ). In this case,

$$
\operatorname{Mor}_{\mathfrak{h}}\left(V, \bigwedge^{2} V\right)=\operatorname{Mor}_{\mathfrak{h}}(V, \mathfrak{h})=\{0\}
$$

(cf. remark after (17)). We have also

$$
\operatorname{Mor}_{\mathfrak{h}}\left(V, \bigwedge^{2} \mathfrak{h}\right)=\{0\}
$$

as a consequence of the following lemma.

Lemma 2.2 Let $o(N)$ denote the orthogonal complex Lie algebra acting in $\mathbb{C}^{N}$. Then

$$
\operatorname{Mor}_{o(N)}\left(\mathbb{C}^{N}, \bigwedge^{2} o(N)\right)=\{0\} \quad \text { for } \quad N \neq 3 .
$$

Proof: It is sufficient to consider $N>3$. We consider two cases.

1. $N=2 n$. Recall that weights of a $\mathfrak{g}$-module are functions on a basis of a Cartan subalgebra of $\mathfrak{g}$. For the $o(N)$-module $\mathbb{C}^{N}$, these functions are non-zero at exactly one point. The basis may be chosen in such a way that the non-zero values of these functions are \pm 1 .

The weights of $o(N) \cong \Lambda^{2} \mathbb{C}^{N}$ are sums of two different weights of $\mathbb{C}^{N}$, hence either they are zero or they are non-zero at exactly two points, where they have value \pm 1 (note that this shows that $\operatorname{Mor}_{o(2 n)}\left(\mathbb{C}^{2 n}, o(2 n)\right)=\{0\}$.)

The weights of $\stackrel{2}{\wedge}^{\prime} o(N)$ are sums of two different weights of $o(N)$. The only weights having one-point support have values \pm 2 , hence there is no nontrivial intertwiner from $\mathbb{C}^{N}$ to $\wedge^{2} o(N)$.

2. $N=2 n+1$. For a suitably chosen $e_{0} \in \mathbb{C}^{N}$, we may identify $o(2 n)$ as the subalgebra of $o(2 n+1)$ stabilizing $e_{0}$, and acting on its orthogonal complement, identified with $\mathbb{C}^{2 n}$ (we can also choose the quadratic form conveniently, if needed). We have

$$
\operatorname{Mor}_{o(2 n+1)}\left(\mathbb{C}^{2 n+1}, \bigwedge^{2}\left(\bigwedge^{2} \mathbb{C}^{2 n+1}\right)\right) \subset \operatorname{Mor}_{o(2 n)}\left(\mathbb{C} \oplus \mathbb{C}^{2 n}, \bigwedge^{2}\left(\bigwedge^{2} \mathbb{C}^{2 n+1}\right)\right)
$$


Since

$$
\begin{gathered}
\bigwedge^{2}\left(\mathbb{C} \oplus \mathbb{C}^{2 n}\right) \cong \mathbb{C}^{2 n} \oplus \bigwedge^{2} \mathbb{C}^{2 n} \\
\bigwedge^{2}\left(\bigwedge^{2}\left(\mathbb{C} \oplus \mathbb{C}^{2 n}\right)\right) \cong \bigwedge^{2} \mathbb{C}^{2 n} \oplus\left(\mathbb{C}^{2 n} \otimes \bigwedge^{2} \mathbb{C}^{2 n}\right) \oplus \bigwedge^{2}\left(\bigwedge^{2} \mathbb{C}^{2 n}\right),
\end{gathered}
$$

and

$$
\begin{gathered}
\operatorname{Mor}_{o(2 n)}\left(\mathbb{C}, \bigwedge^{2} \mathbb{C}^{2 n}\right)=\operatorname{Mor}_{o(2 n)}(\mathbb{C}, o(2 n))=(o(2 n))_{o(2 n)}=\{0\}, \\
\operatorname{Mor}_{o(2 n)}\left(\mathbb{C}, \mathbb{C}^{2 n} \otimes o(2 n)\right) \cong \operatorname{Mor}_{o(2 n)}\left(\mathbb{C}^{2 n}, o(2 n)\right)=\{0\}, \\
\operatorname{Mor}_{o(2 n)}\left(\mathbb{C}, \bigwedge^{2} o(2 n)\right) \cong\left(\bigwedge^{2} o(2 n)\right)_{o(2 n)}=\{0\},
\end{gathered}
$$

we have

$$
\operatorname{Mor}_{o(2 n)}\left(\mathbb{C} \oplus \mathbb{C}^{2 n}, \bigwedge^{2} o(N)\right) \cong \operatorname{Mor}_{o(2 n)}\left(\mathbb{C}^{2 n}, \bigwedge^{2} o(N)\right) .
$$

If $T: \mathbb{C}^{N} \rightarrow \bigwedge^{2} o(N)$ is a nonzero $o(N)$-morphism, then $\left.f\right|_{\mathbb{C}}=0$ and $\left.f\right|_{\mathbb{C}^{2 n}}$ is injective (since $\mathbb{C}^{2 n}$ is $o(2 n)$-irreducible). Let $X$ be any element of $o(N)$ which applied to $e_{0}$ gives a non-zero element of $\mathbb{C}^{2 n}$ (for instance $X=\Omega_{j 0}$ ). We obtain the contradiction

$$
0 \neq T\left(X e_{0}\right)=X T\left(e_{0}\right)=X(0)=0,
$$

showing that $T$ has to be zero.

Due to (19), (20) and (田),

$$
\text { Q.E.D. }
$$

$$
\operatorname{Mor}_{\mathfrak{h}}\left(V, \bigwedge^{2} \mathfrak{g}\right)=\operatorname{Mor}_{\mathfrak{h}}(V, V \otimes \mathfrak{h})
$$

Using the fact that $\mathfrak{h}$-modules $V$ and $\mathfrak{h}$ are isomorphic to their duals, we have

$$
\operatorname{Mor}_{\mathfrak{h}}(V, V \otimes \mathfrak{h}) \cong \operatorname{Mor}_{\mathfrak{h}}(\mathfrak{h}, V \otimes V) \cong \operatorname{Mor}_{\mathfrak{h}}\left(\mathfrak{h}, \bigwedge^{2} V\right)
$$

Here in the last equality we have used the following simple fact (which may be easily proved using e.g. [8], § 7, Prop. 10).

Lemma 2.3 $\operatorname{Mor}_{o(N)}\left(o(N), \mathbb{C}^{N} \otimes_{\text {symm }} \mathbb{C}^{N}\right)=\{0\} \quad$ for $N>2$.

(Here the subscript 'symm' refers to the symmetric part). It follows that

$$
\operatorname{Mor}_{\mathfrak{h}}(V, \bigwedge \mathfrak{2} \mathfrak{g}) \cong \operatorname{Mor}_{\mathfrak{h}}(\mathfrak{h}, \mathfrak{h}) \cong \begin{cases}\mathbb{R}^{2} & \text { if } p+q=4 \\ \mathbb{R} & \text { otherwise. }\end{cases}
$$

The identity of $\mathfrak{h}$ defines the following element $F_{0}$ of $\operatorname{Mor}_{\mathfrak{h}}(V, V \otimes \mathfrak{h})$ :

$$
V \ni x \mapsto F_{0}(x):=g^{j k} e_{j} \otimes \Omega_{x, e_{k}} \in V \otimes \mathfrak{h}
$$


( $g^{j k}$ is the contravariant metric). When $p+q=4$, the Hodge star operation $*: \mathfrak{h} \rightarrow \mathfrak{h}$ given by

$$
\left.\left.* \Omega_{x, z}:=g(x) \wedge g(z)\right\lrcorner \mathrm{Vol}, \quad\left(* \Omega_{x, z}\right) y==g(x) \wedge g(y) \wedge g(z)\right\lrcorner \mathrm{Vol}=x \times y \times z
$$

(Vol is the volume element, $\times$ denotes the vector product of three vectors) intertwines $\mathfrak{h}$ with itself and is not proportional to the identity. It defines another, linearly independent from $F_{0}$, intertwiner from $V$ to $V \otimes \mathfrak{h}$ :

$$
F_{1}:=(\mathrm{id} \otimes *) \circ F_{0} .
$$

Note that an element $F \in \operatorname{Mor}_{\mathfrak{h}}(V, \stackrel{2}{\wedge} \mathfrak{g})$ belongs to $Z(V, \stackrel{2}{\wedge} \mathfrak{g})$ if and only if the map

$$
V \times V \ni(x, y) \mapsto x F(y) \in \bigwedge^{2} \mathfrak{g}
$$

is symmetric. It is easily checked that $x F_{0}(y)$ is antisymmetric:

$$
x F_{0}(y)=y \wedge x .
$$

If $p+q \neq 4$, it means that

$$
Z\left(V, \bigwedge^{2} \mathfrak{g}\right) \cap \operatorname{Mor}_{\mathfrak{h}}\left(V, \bigwedge^{2} \mathfrak{g}\right)=\{0\}
$$

If $p+q=4$, one can show that

$$
x F_{1}(y)=g^{j k} e_{j} \wedge\left(x \times y \times e_{k}\right),
$$

which is also antisymmetric and linearly independent from (29). This shows that (30) holds also in this case.

Now let us consider the case $p+q=3$. Since $V \cong \bigwedge^{2} V \cong \mathfrak{h}$, we have

$$
\operatorname{Mor}_{\mathfrak{h}}\left(V, \bigwedge^{2} V\right) \cong \mathbb{R}, \quad \operatorname{Mor}_{\mathfrak{h}}(V, V \otimes \mathfrak{h}) \cong \mathbb{R}, \quad \operatorname{Mor}_{\mathfrak{h}}\left(V, \bigwedge^{2} \mathfrak{h}\right) \cong \mathbb{R}
$$

(cf. also (23)). Note that the symmetry of (28) for $F \in \operatorname{Mor}_{\mathfrak{h}}(V, \stackrel{2}{\wedge} \mathfrak{g})$ means the symmetry condition separately for each of its three components (in the decomposition (4) with $\mathfrak{n}=V$ ). The first component is proportional to

$$
V \ni x \mapsto T(x):=g(x)\lrcorner \mathrm{Vol} \in \bigwedge^{2} V .
$$

The symmetry is trivially satisfied in this case. The second component, proportional to (25) satisfies the symmetry of (28) if and only if it is zero, by (29). The third component is proportional to

$$
(\Omega \otimes \Omega)(T \otimes T) T .
$$

One can show by a direct calculation, that (33) does not satisfy the symmetry condition. We conclude that in the case when $p+q=3$,

$$
Z\left(V, \bigwedge^{2} \mathfrak{g}\right) \cap \operatorname{Mor}_{\mathfrak{h}}\left(V, \bigwedge^{2} \mathfrak{g}\right)=\{\mathbb{R} \cdot T\}
$$


But $T=-\frac{1}{2} \partial s$, where $s$ is given by (18). This ends the proof of the theorem.

Q.E.D.

In view of this theorem, the classification of Lie bialgebra structures on $\mathfrak{g}=V \rtimes \mathfrak{h}$ consists in a description of equivalence classes (modulo Aut $\mathfrak{g}$ ) of $r \in \bigwedge^{2} \mathfrak{g}$ such that $[r, r] \in$ $\left(\bigwedge^{3} \mathfrak{g}\right)_{\mathfrak{g}}$

Each $r \in \bigwedge^{2} \mathfrak{g}$ has a decomposition

$$
r=a+b+c,
$$

corresponding to the decomposition (5)

$$
\bigwedge^{2} \mathfrak{g}=\bigwedge^{2} V \oplus(V \wedge \mathfrak{h}) \oplus \bigwedge^{2} \mathfrak{h}
$$

We have also the following decomposition of the Schouten bracket

$$
[r, r]=2[a, b]+(2[a, c]+[b, b])+2[b, c]+[c, c],
$$

corresponding to the decomposition

$$
\bigwedge^{3} \mathfrak{g}=\bigwedge^{3} V \oplus\left(\bigwedge^{2} V \wedge \mathfrak{h}\right) \oplus\left(V \wedge \bigwedge^{2} \mathfrak{h}\right) \oplus \bigwedge^{3} \mathfrak{h}
$$

Note that

$$
\left(\bigwedge^{3} \mathfrak{g}\right)_{\mathfrak{g}}=\left(\bigwedge^{3} V\right)_{\mathfrak{g}} \oplus\left(\bigwedge^{2} V \wedge \mathfrak{h}\right)_{\mathfrak{g}} \oplus\left(V \wedge \bigwedge^{2} \mathfrak{h}\right)_{\mathfrak{g}} \oplus\left(\bigwedge^{3} \mathfrak{h}\right)_{\mathfrak{g}}
$$

We shall show that this space is one-dimensional for $p+q>3$. Note that the isomorphism $\Omega$ defines a canonical $\mathfrak{h}$-invariant element of $\left({ }^{2} V\right)^{*} \otimes \mathfrak{h}$, or, using the identification of $V$ and $V^{*}$, a canonical $\mathfrak{h}$-invariant element of $\Lambda^{2} V \otimes \mathfrak{h}$. We shall denote this element again by $\Omega$. It is given by

$$
\Omega=g^{j l} g^{k m} e_{j} \wedge e_{k} \otimes \Omega_{l, m}
$$

(in any basis). This element is also $V$-invariant:

$$
x \Omega=-g^{j l} g^{k m} e_{j} \wedge e_{k} \wedge\left(e_{l} x_{m}-e_{m} x_{l}\right)=0 \quad \text { for } x \in V .
$$

Theorem 2.4 If $\operatorname{dim} V>3$ then $\left(\bigwedge^{3} \mathfrak{g}\right)_{\mathfrak{g}}=\left(\stackrel{2}{\Lambda}^{2} \wedge \mathfrak{h}\right)_{\mathfrak{g}}=\mathbb{R} \cdot \Omega$.

Proof: We calculate all terms in (36).

1. If $w \in \Lambda^{3} V$ is $\mathfrak{h}$-invariant, then

$$
V \ni x \mapsto g(x)\lrcorner w \in \bigwedge^{2} V
$$

belongs to $\operatorname{Mor}_{\mathfrak{h}}(V, \stackrel{2}{\wedge} V)$. From (19) it follows that $w=0$. Hence $\left(\stackrel{3}{\Lambda}^{\prime}\right)_{\mathfrak{g}}=\{0\}$. 
2. The second component in (36) is contained in (24). We already know that $\Omega$ is $\mathfrak{g}$ invariant. If $p+q=4$, the second (linearly independent) $\mathfrak{h}$-invariant element (id $\otimes *$ ) $\Omega$

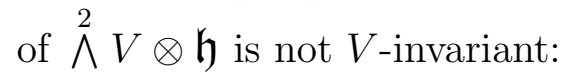

$x($ id $\left.\left.\otimes *) \Omega=-g^{j l} g^{k m} e_{j} \wedge e_{k} \wedge\left(* \Omega_{l m}\right) x=e_{j} \wedge e_{k} \wedge\left(e^{j} \wedge e^{k} \wedge g(x)\right\lrcorner \operatorname{Vol}\right)=2 g(x)\right\lrcorner \operatorname{Vol}$.

It follows that $(\stackrel{2}{\wedge} V \wedge \mathfrak{h})_{\mathfrak{g}}=\mathbb{R} \cdot \Omega$.

3. The third component in (36) is zero by (20).

4. We shall show that $\left(\bigwedge^{3} \mathfrak{h}\right)_{V}=\{0\}$. If $w \in \bigwedge^{3} \mathfrak{h}$ is $V$-invariant, then

$$
0=x w \in V \wedge \bigwedge^{2} \mathfrak{h} \quad \text { for } x \in V
$$

hence

$$
0=\xi\lrcorner x w \quad \text { for } x \in V, \xi \in V^{*} .
$$

Since $\left.\xi\lrcorner x w=-\omega_{\xi, x}\right\lrcorner w$, where $\omega_{\xi, x} \in \mathfrak{h}^{*}$ is defined by $\omega_{\xi, x}(A):=\langle\xi, A x\rangle$, we have

$$
\alpha\lrcorner w=0 \quad \text { for } \alpha \in \mathfrak{h}^{*}
$$

(elements of the form $\omega_{\xi, x}$ span $\mathfrak{h}^{*}$ ), hence $w=0$.

Q.E.D.

From this result and (35) it follows that Lie bialgebra structures on $\mathfrak{g}$ are (for $p+q>3$ ) in one-to-one correspondence with $r=a+b+c \in \bigwedge^{2} \mathfrak{g}$ such that

$$
\begin{aligned}
{[c, c] } & =0 \\
{[b, c] } & =0 \\
2[a, c]+[b, b] & =t \Omega \quad(t \in \mathbb{R}) \\
{[a, b] } & =0 .
\end{aligned}
$$

Equation (38) means that $c$ is a triangular $r$-matrix on $\mathfrak{h}$ (this is the semi-classical counterpart of a known theorem [9] excluding the case when the homogeneous part $H$ is $q$-deformed). Equation (39) tells that $b$, as a map from $\mathfrak{h}^{*}$ to $V$, is a cocycle:

$$
b\left([\alpha, \beta]_{c}\right)=c(\alpha) b(\beta)-c(\beta) b(\alpha) \quad \text { for } \alpha, \beta \in \mathfrak{h}^{*},
$$

the Lie bracket on $\mathfrak{h}^{*}$ being defined by the triangular $c \in \bigwedge^{2} \mathfrak{h}$ as in (8):

$$
[\alpha, \beta]_{c}=c(\alpha) \beta-c(\beta) \alpha
$$

and the action of $\mathfrak{h}^{*}$ on $V$ is defined using the homomorphism from $\mathfrak{h}^{*}$ to $\mathfrak{h}$ given by $c$ :

$$
c(\alpha):=\alpha\lrcorner c \in \mathfrak{h} \quad \text { for } \alpha \in \mathfrak{h}^{*}
$$

(as in (10)). To get (42) one can use (11) with $\alpha, \beta \in \mathfrak{h}^{*}, \gamma \in V^{*}$.

Here are some particular solutions of (38)-(41). 
1. $a=0, b=0, c \in \stackrel{2}{\wedge} \mathfrak{h}$ triangular.

2. $b=0, c=0, a \in \bigwedge^{2} V$ arbitrary. This type of solutions we call 'soft deformations' [10.

3. $a=0, c=0,[b, b]=t \Omega$. There is a family of solutions of the latter equation, parameterized by vectors in $V$. Namely, for each $x \in V$,

$$
\left.b_{x}:=F_{0}(x)=g^{j k} e_{j} \otimes \Omega_{x, e_{k}}=\frac{1}{2} g(x)\right\lrcorner \Omega
$$

satisfies this equation with $t=-g(x, x)\left(F_{0}\right.$ is defined in (25)). Moreover, since $\left[x, b_{x}\right]=0$ (easy calculation),

$$
b=b_{x}+x \wedge X, \quad X \in \mathfrak{h}_{x} \quad(\text { stabilizer of } x \text { in } \mathfrak{h})
$$

satisfies $[b, b]=\left[b_{x}, b_{x}\right]=-g(x, x) \Omega$. Indeed, $[x \wedge X, x \wedge X]=0$ and

$$
\left[x \wedge X, b_{x}\right]=x \wedge X b_{x}-X \wedge\left[x, b_{x}\right]=x \wedge b_{X x}=0 .
$$

Note the following two properties of $b$ given in (45) for $x \neq 0$ :

$$
\begin{gathered}
{[a, b]=0 \Longleftrightarrow(X-2) a \in x \wedge V \quad \text { for } a \in \bigwedge^{2} V} \\
(-v) b=x \wedge(X-1) v \quad \text { for } v \in V
\end{gathered}
$$

(the first follows from $[a, b]=x \wedge(X-2) a)$.

Proposition 2.5 Suppose $b$ is given by $(45)$ with $x \neq 0$. If $X$ has no eigenvalue 1 on $V$ and no eigenvalue 2 on $\bigwedge^{2} V$, then for any solution a of (41), $r=a+b$ can be transformed to $b$ by a suitable internal automorphism of $\mathfrak{g}$.

Proof: Since $X$ preserves $x \wedge V$ and $(X-2)$ is invertible on $\stackrel{2}{\wedge} V$, the right hand side of (46) is equivalent to $a \in x \wedge V$. Since $X-1$ is invertible, $(-v) b$ runs over $x \wedge V$ when $v$ runs over $V$. It follows that $\left(\mathrm{Ad}_{-v} \otimes \mathrm{Ad}_{-v}\right)(a+b)=(a+(-v) b)+b$ is equal $b$ for some $v \in V$ (see also (55)).

Of course, a generic $X$ will satisfy the assumptions of the above proposition.

Q.E.D.

\section{The case of the Poincaré group}

We now fix $(p, q)=(1,3)$. It means that $V \cong \mathbb{R}^{1+3}$ is the four-dimensional Minkowski space-time, $\mathfrak{h}=o(1,3) \cong \operatorname{sl}(2, \mathbb{C})$ is the Lorentz Lie algebra and $\mathfrak{g}$ is the Poincaré Lie algebra.

We are interested in classifying the solutions of (38)-(41) up to the automorphisms of $\mathfrak{g}$. In particular, $c$ can be always chosen to be a normal form of a triangular classical $r$-matrix on the Lorentz Lie algebra, as listed in [11]. In the next section, for each such non-zero $c$, 
we shall solve (39)-(41) completely (up to an automorphism). Moreover, we shall find all solutions with $c=0$ provided $t=0$. The results are shown in Table 1 below. Let us explain the notation. We introduce the standard generators of $\mathfrak{h}=\operatorname{sl}(2, \mathbb{C})$ :

$$
H=\frac{1}{2}\left[\begin{array}{cc}
1 & 0 \\
0 & -1
\end{array}\right], \quad X_{+}=\left[\begin{array}{ll}
0 & 1 \\
0 & 0
\end{array}\right], \quad X_{-}=\left[\begin{array}{ll}
0 & 0 \\
1 & 0
\end{array}\right] .
$$

The action of $X \in \operatorname{sl}(2, \mathbb{C})$ on a vector $v \in V$ is given by $X(v):=X v+v X^{+}$, the space $V$ being identified with the set of hermitian $2 \times 2$ matrices, where $X^{+}$is the hermitian conjugate of $X$. We fix the Lorentz basis $e_{0}, e_{1}, e_{2}, e_{3}$ in $V$ given by the standard Pauli matrices:

$$
e_{0}=\left[\begin{array}{ll}
1 & 0 \\
0 & 1
\end{array}\right], \quad e_{1}=\sigma_{1}=\left[\begin{array}{ll}
0 & 1 \\
1 & 0
\end{array}\right], \quad e_{2}=\sigma_{2}=\left[\begin{array}{cc}
0 & -i \\
i & 0
\end{array}\right], \quad e_{3}=\sigma_{3}=\left[\begin{array}{cc}
1 & 0 \\
0 & -1
\end{array}\right]
$$

We denote by $J$ the multiplication by the imaginary unit in $\mathfrak{h}$. As acting on $V$, the basic generators of $\mathfrak{h}$ are given by

$$
\begin{gathered}
H=L_{3}=\Omega_{30}=e_{0} \otimes e^{3}+e_{3} \otimes e^{0}, \quad J H=-M_{3}=\Omega_{21}=e_{1} \otimes e^{2}-e_{2} \otimes e^{1} \\
X_{+}=\Omega_{10}+\Omega_{13}=\Omega_{e_{1}, e_{+}}, \quad J X_{+}=\Omega_{02}+\Omega_{32}=\Omega_{e_{+}, e_{2}}, \\
X_{-}=\Omega_{10}+\Omega_{31}=\Omega_{e_{1}, e_{-}}, \quad J X_{-}=\Omega_{20}+\Omega_{32}=\Omega_{e_{2}, e_{-}} .
\end{gathered}
$$

It is also convenient to introduce the light-cone vectors $e_{ \pm}:=e_{0} \pm e_{3}$.

The table lists 21 cases labelled by the number $\mathrm{N}$ in the last column. In the forth column (labelled by \#) we indicate the number of essential parameters (more precisely the maximal number of such parameters) involved in the deformation. This number is in many cases less than the number of parameters actually occurring in the table. The final reduction of the number of parameters can be achieved using two following one-parameter groups of automorphisms of $\mathfrak{g}$ :

1. the group of dilations: $(v, X) \mapsto(\lambda v, X)$ (in cases $1,2,3,4,6)$,

2. the group of internal automorphisms generated by $H$ and the group of dilations (in cases $11,12,15,17,18)$.

(the table looks more concise before the final reduction).

Remark 3.1 The table below differs a little from the table announced in [1]. Some errors are corrected and the presentation is improved. Solutions (51), (53) are now presented separately (they are not included in the table: they form the known part of the not yet solved problem $[b, b]=t \Omega, t \neq 0$ ) and are now supplemented by (54). 


\begin{tabular}{|c|c|c|c|}
\hline$c$ & $b$ & $a$ & $\# \mathrm{~N}$ \\
\hline$\gamma J H \wedge H$ & $\overline{0}$ & $\overline{\alpha \alpha e_{+} \wedge e_{-}+\widetilde{\alpha} e_{1} \wedge e_{2}}$ & \begin{tabular}{|l|l|}
2 & 1 \\
\end{tabular} \\
\hline \multirow[t]{3}{*}{$J X_{+} \wedge X_{+}$} & $\beta_{1} b_{e_{+}}+\beta_{2} e_{+} \wedge J H$ & 0 & \begin{tabular}{|l|l|}
1 & 2 \\
\end{tabular} \\
\hline & $\beta b_{e_{+}}$ & $\alpha e_{+} \wedge e_{1}$ & \begin{tabular}{|l|l|}
1 & 3 \\
\end{tabular} \\
\hline & $\beta\left(e_{1} \wedge X_{+}+e_{2} \wedge J X_{+}\right)$ & $e_{+} \wedge\left(\alpha_{1} e_{1}+\alpha_{2} e_{2}\right)-\beta^{2} e_{1} \wedge e_{2}$ & \begin{tabular}{|l|l|}
2 & 4 \\
\end{tabular} \\
\hline $\begin{array}{c}H \wedge X_{+}- \\
J H \wedge J X_{+}+ \\
\gamma J X_{+} \wedge X_{+}\end{array}$ & 0 & 0 & $10 \mid 5$ \\
\hline$H \wedge X_{+}$ & $\beta_{1} b_{e_{2}}+\beta_{2} e_{2} \wedge X_{+}$ & 0 & \begin{tabular}{|l|l|}
1 & 6 \\
\end{tabular} \\
\hline \multirow[t]{15}{*}{0} & $b_{e_{+}}+\beta e_{+} \wedge J H$ & 0 & \begin{tabular}{|l|l|}
1 & 7 \\
\end{tabular} \\
\hline & $b_{e_{+}}+\beta e_{+} \wedge X_{+}$ & 0 & \begin{tabular}{|l|l|}
1 & 8 \\
\end{tabular} \\
\hline & $\begin{aligned} e_{1} & \wedge\left(X_{+}+\beta J X_{+}\right)+ \\
+e_{+} & \wedge\left(H+\sigma X_{+}\right), \quad \sigma=0, \pm 1\end{aligned}$ & $\alpha e_{+} \wedge e_{2}$ & \begin{tabular}{|l|l|}
2 & 9 \\
\end{tabular} \\
\hline & $e_{1} \wedge J X_{+}+e_{+} \wedge X_{+}$ & $\alpha_{1} e_{-} \wedge e_{1}+\alpha_{2} e_{+} \wedge e_{2}$ & \begin{tabular}{|l|l|}
2 & 10 \\
\end{tabular} \\
\hline & $e_{2} \wedge X_{+}$ & $\alpha_{1} e_{+} \wedge e_{1}+\alpha_{2} e_{-} \wedge e_{2}$ & \begin{tabular}{|l|l|}
1 & 11 \\
\end{tabular} \\
\hline & $e_{+} \wedge X_{+}$ & $e_{-} \wedge\left(\alpha e_{+}+\alpha_{1} e_{1}+\alpha_{2} e_{2}\right)+\widetilde{\alpha} e_{+} \wedge e_{2}$ & \begin{tabular}{|l|l|}
3 & 12 \\
\end{tabular} \\
\hline & $e_{0} \wedge J H$ & $\alpha_{1} e_{0} \wedge e_{3}+\alpha_{2} e_{1} \wedge e_{2}$ & \begin{tabular}{|l|l|}
2 & 13 \\
\end{tabular} \\
\hline & $e_{3} \wedge J H$ & $\alpha_{1} e_{0} \wedge e_{3}+\alpha_{2} e_{1} \wedge e_{2}$ & 214 \\
\hline & $e_{+} \wedge J H$ & $\alpha_{1} e_{0} \wedge e_{3}+\alpha_{2} e_{1} \wedge e_{2}$ & \begin{tabular}{l|l|l|}
1 & 15 \\
\end{tabular} \\
\hline & $e_{1} \wedge H$ & $\alpha_{1} e_{0} \wedge e_{3}+\alpha_{2} e_{1} \wedge e_{2}$ & \begin{tabular}{|l|l|}
2 & 16 \\
\end{tabular} \\
\hline & $e_{+} \wedge H$ & $\alpha e_{1} \wedge e_{2}+\alpha_{1} e_{+} \wedge e_{1}$ & \begin{tabular}{|l|l|}
1 & 17 \\
\end{tabular} \\
\hline & $e_{+} \wedge(H+\beta J H)$ & $\alpha e_{1} \wedge e_{2}$ & \begin{tabular}{l|l|}
1 & 18 \\
\end{tabular} \\
\hline & \multirow[t]{3}{*}{0} & $e_{1} \wedge e_{+}$ & \begin{tabular}{|l|l|}
0 & 19 \\
\end{tabular} \\
\hline & & $e_{1} \wedge e_{2}$ & \begin{tabular}{|l|l|l|}
0 & 20 \\
\end{tabular} \\
\hline & & $e_{0} \wedge e_{3}+\alpha e_{1} \wedge e_{2}$ & \begin{tabular}{|l|l|}
1 & 21 \\
\end{tabular} \\
\hline
\end{tabular}

Table 1: Normal forms of $r$ for $c \neq 0$ or $t=0$.

In the case when $c=0$ and $t \neq 0$, the only solutions we know are based on formula (45). We describe them now. We shall use yet another standard generators of $\mathfrak{h}$ :

$$
M_{i}=\varepsilon_{i j k} e_{k} \otimes e^{j}, \quad L_{i}=e_{0} \otimes e^{i}+e_{i} \otimes e^{0} \quad(i, j, k=1,2,3) .
$$

If we set $x:=e_{0}$ in (44), we obtain

$$
b_{e_{0}}=e_{1} \wedge L_{1}+e_{2} \wedge L_{2}+e_{3} \wedge L_{3},
$$

which is the known [12] classical $r$-matrix corresponding to so called $\kappa$-deformation. More generally, using (45), we have

$$
b=b_{e_{0}}+\lambda e_{0} \wedge M_{3}
$$

(any element of $\mathfrak{h}_{x} \cong o(0,3)$ can be rotated to $\left.\lambda M_{3}\right)$. Since $M_{3}$ has only imaginary eigenvalues, adding $a$ we do not obtain essentially different solutions, cf. Prop. 2.5.

Taking $x=e_{1}$ in (44), we obtain another solution

$$
b_{e_{1}}=e_{0} \wedge L_{1}-e_{2} \wedge M_{3}+e_{3} \wedge M_{2}
$$


(this one is $M_{1}, L_{2}, L_{3}$-invariant). There are three types of elements in $\mathfrak{h}_{x} \cong o(1,2)$, according to the sign of the Killing form. We have thus three types of perturbations (45) of (52):

$$
b=b_{e_{1}}+\lambda e_{1} \wedge Y, \quad Y=M_{1} \text { or } Y=M_{1}+L_{3} \text { or } Y=L_{3}=H .
$$

In the first two cases, adding a does not yield new solutions, since $M_{1}$ has only imaginary eigenvalues and $M_{1}+L_{3}$ is nilpotent. Since non-zero eigenvalues of $H$ are \pm 1 , adding $a$ in the third case we can obtain a nontrivial modification when $\lambda= \pm 1, \pm 2$. We obtain then the following four families of solutions:

$$
b=b_{e_{1}} \pm k e_{1} \wedge H+\alpha e_{k} \wedge e_{ \pm}, \quad k=1,2
$$

(using the automorphisms generated by $H$, we can assume that $\alpha= \pm 1$ ).

\section{The proof for $c \neq 0$}

The four types of non-zero triangular $c$ in the table above are taken from [11]. We consider each case separately. We denote by $\left(H^{*}, J H^{*}, X_{ \pm}^{*}, J X_{ \pm}^{*}\right)$ the basis dual to $\left(H, J H, X_{ \pm}, J X_{ \pm}\right)$.

\section{$4.1 c=J H \wedge H$}

First we calculate brackets (43) of basis elements and write down corresponding cocycle condition (42). We do not consider pairs of elements from the subset $\left\{X_{+}^{*}, J X_{+}^{*}, X_{-}^{*}, J X_{-}^{*}\right\}$, since for them the corresponding condition (42) is trivial. We have

$$
\begin{aligned}
& {\left[J H^{*}, H^{*}\right]_{c}=0} \\
& {\left[X_{ \pm}^{*}, H^{*}\right]_{c}= \pm J X_{ \pm}^{*}} \\
& {\left[J X_{ \pm}^{*}, H^{*}\right]_{c}=\mp X_{ \pm}^{*}} \\
& {\left[X_{ \pm}^{*}, J H^{*}\right]_{c}= \pm X_{ \pm}^{*}} \\
& {\left[J X_{ \pm}^{*}, J H^{*}\right]_{c}= \pm J X_{ \pm}^{*}} \\
& \begin{aligned}
0 & =H b\left(H^{*}\right)+J H b\left(J H^{*}\right) \\
b\left(J X_{ \pm}^{*}\right) & = \pm J H b\left(X_{ \pm}^{*}\right) \\
b\left(X_{ \pm}^{*}\right) & =\mp J H b\left(J X_{ \pm}^{*}\right) \\
b\left(X_{ \pm}^{*}\right) & =\mp H b\left(X_{ \pm}^{*}\right) \\
b\left(J X_{ \pm}^{*}\right) & =\mp H b\left(J X_{ \pm}^{*}\right) .
\end{aligned}
\end{aligned}
$$

Due to (48), $H(V) \cap J H(V)=\{0\}$, hence the last four formulas imply

$$
b\left(X_{ \pm}^{*}\right)=0, \quad b\left(J X_{ \pm}^{*}\right)=0 .
$$

For the same reason, the first equation,

$$
H b\left(H^{*}\right)=-J H b\left(J H^{*}\right),
$$

has the obvious solution $b\left(H^{*}\right) \in \operatorname{ker} H, b\left(J H^{*}\right) \in \operatorname{ker} J H$, which can be written as follows

$$
b\left(H^{*}\right)=J H(v), \quad b\left(J H^{*}\right)=-H(v), \quad v \in V,
$$

or

$$
b=H \wedge J H(v)-J H \wedge H(v)=v c .
$$

Using the internal automorphism $\mathrm{Ad}_{-v}=\mathrm{id}-v$ of $\mathfrak{g}$, we can transform $r=a+b+c$ into

$$
\left(\mathrm{Ad}_{-v} \otimes \mathrm{Ad}_{-v}\right) r=r+(-v) r+(v \otimes v) r=(a+(-v) b+(v \otimes v) c)+(b+(-v) c)+c
$$


hence we can always set $b=0$.

The last equation to solve is (40) with $b=0$. Since $\Omega$ represents the isomorphism of $\wedge^{2} V$ and $\mathfrak{h}$, it has rank equal $6=\operatorname{dim} \mathfrak{h}$ (as an element of the tensor product of $\stackrel{2}{\Lambda}^{2}$ and $\mathfrak{h})$. The Schouten bracket $[c, a]$ is a tensor of rank at most 2, because

$$
[J H \wedge H, a]=J H \wedge[H, a]-H \wedge[J H, a] .
$$

It follows that $t=0$, hence equation (40) reduces to $[c, a]=0$, i.e.

$$
[H, a]=0, \quad[J H, a]=0 .
$$

It is clear that $a \in \AA^{2} V$, considered as an element of $\mathfrak{h}$ has to be a combination of $H$ and

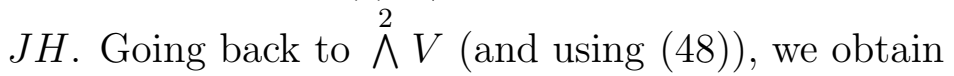

$$
a=\lambda e_{0} \wedge e_{3}+\mu e_{1} \wedge e_{2}, \quad \lambda, \mu \in \mathbb{R} .
$$

Since we can multiply our solution $r=c+a$ by any number, we obtain the first case of the table.

It is easy to check that $X \in \mathfrak{h}$ and $X c \equiv[X, c]=0$ implies that $X$ is a combination of $H$ and $J H$. Such $X$ gives rise to a group of internal automorphisms of $\mathfrak{g}$, leaving $c$ invariant. These automorphisms leave invariant also $a$, hence they cannot be used to a further reduction of $a$.

\section{$4.2 c=J X_{+} \wedge X_{+}$}

First we calculate the brackets (formula (43)) of basis elements (only those contributing to the cocycle condition):

$$
\begin{aligned}
{\left[J X_{+}^{*}, X_{+}^{*}\right]_{c} } & =2 H^{*} \\
{\left[J X_{+}^{*}, H^{*}\right]_{c} } & =-2 X_{-}^{*} \\
{\left[X_{+}^{*}, H^{*}\right]_{c} } & =-2 J X_{-}^{*} \\
{\left[X_{+}^{*}, J H^{*}\right]_{c} } & =2 X_{-}^{*} \\
{\left[J X_{+}^{*}, J H^{*}\right]_{c} } & =-2 J X_{-}^{*}
\end{aligned}
$$

and $X_{-}^{*}, J X_{-}^{*}$ are central elements. It follows that the cocycle condition (42) reads

$$
\begin{aligned}
2 b\left(H^{*}\right) & =X_{+} b\left(X_{+}^{*}\right)+J X_{+} b\left(J X_{+}^{*}\right) \\
2 b\left(X_{-}^{*}\right) & =-X_{+} b\left(H^{*}\right) \\
2 b\left(J X_{-}^{*}\right) & =J X_{+} b\left(H^{*}\right) \\
2 b\left(X_{-}^{*}\right) & =-J X_{+} b\left(J H^{*}\right) \\
2 b\left(J X_{-}^{*}\right) & =-X_{+} b\left(J H^{*}\right)
\end{aligned}
$$

and $b\left(X_{-}^{*}\right), b\left(J X_{-}^{*}\right)$ are $X_{+}$and $J X_{+}$-invariant (the latter property is already a consequence of (64)-(65), since $X_{+} \circ J X_{+}=0=J X_{+} \circ X_{+}$). We recall (cf. (49)) that

$$
X_{+} x=2 x^{-} e_{1}+x^{1} e_{+}, \quad J X_{+} x=-2 x^{-} e_{2}-x^{2} e_{+} \quad \text { for } x=x^{+} e_{+}+x^{-} e_{-}+x^{1} e_{1}+x^{2} e_{2} .
$$


To solve (61)-(65) we can just set $b\left(X_{+}^{*}\right)=x, b\left(J X_{+}^{*}\right)=y$, where $x, y \in V$ are arbitrary vectors and then

$$
\begin{aligned}
b\left(H^{*}\right) & =\frac{1}{2}\left(X_{+} x+J X_{+} y\right)=x^{-} e_{1}-y^{-} e_{2}+\frac{1}{2}\left(x^{1}-y^{2}\right) e_{+} \\
b\left(X_{-}^{*}\right) & =-\frac{1}{2} X_{+} b\left(H^{*}\right)=-\frac{1}{4}\left(X_{+}\right)^{2} x=-\frac{1}{2} x^{-} e_{+} \\
b\left(J X_{-}^{*}\right) & =\frac{1}{2} J X_{+} b\left(H^{*}\right)=\frac{1}{4}\left(J X_{+}\right)^{2} y=\frac{1}{2} y^{-} e_{+} .
\end{aligned}
$$

Equations (64)-(65) will be satisfied by $b\left(J H^{*}\right)=: z$ if

$$
\begin{aligned}
x^{-} e_{+} & =J X_{+} z=-2 z^{-} e_{2}-z^{2} e_{+} \\
-y^{-} e_{+} & =X_{+} z=2 z^{-} e_{1}+z^{1} e_{+},
\end{aligned}
$$

i.e. $z=z^{+} e_{+}-y^{-} e_{1}-x^{-} e_{2}$ with arbitrary $z^{+} \in \mathbb{R}$. We have thus solved (39) completely (the solution is parameterized by $x, y \in V$ and $z^{+} \in \mathbb{R}$ ).

Now we are going to solve (40). Using formula (37) with the basis $e_{+}, e_{-}, e_{1}, e_{2}$, we have

$$
\Omega=e_{-} \wedge e_{+} \otimes H-2 e_{1} \wedge e_{2} \otimes J H+e_{-} \wedge e_{1} \otimes X_{+}+e_{2} \wedge e_{-} \otimes J X_{+}+e_{+} \wedge e_{1} \otimes X_{-}+e_{+} \wedge e_{2} \otimes J X_{-} .
$$

We shall compute terms on the left hand side of (40) which are proportional to $e_{-} \wedge e_{1} \otimes X_{+}$, $e_{-} \wedge e_{2} \otimes X_{+}, e_{2} \wedge e_{-} \otimes J X_{+}$. Note that they may come only from $[b, b]$. Indeed, $\left[X_{+}, a\right]$ and $\left[J X_{+}, a\right]$ are combinations of $e_{+} \wedge e_{1}, e_{+} \wedge e_{2}, e_{+} \wedge e_{-}, e_{1} \wedge e_{2}$, while

$$
[c, a]=J X_{+} \wedge\left[X_{+}, a\right]-X_{+} \wedge\left[J X_{+}, a\right]
$$

Using the general form of $b$,

$b=H \wedge b\left(H^{*}\right)+J H \wedge b\left(J H^{*}\right)+X_{+} \wedge b\left(X_{+}^{*}\right)+J X_{+} \wedge b\left(J X_{+}^{*}\right)+X_{-} \wedge b\left(X_{-}^{*}\right)+J X_{-} \wedge b\left(J X_{-}^{*}\right)$, it is clear that the terms in $[b, b]$ which contain $X_{+}$are the following:

$$
\begin{gathered}
2\left(\left[H, X_{+}\right] \wedge b\left(H^{*}\right) \wedge b\left(X_{+}^{*}\right)+\left[J X_{+}, J H\right] \wedge b\left(J X_{+}^{*}\right) \wedge b\left(J H^{*}\right)\right)+2 X_{+} \wedge\left[b\left(X_{+}^{*}\right), b\right]= \\
=2 X_{+} \wedge\left(b\left(H^{*}\right) \wedge b\left(X_{+}^{*}\right)+b\left(J X_{+}^{*}\right) \wedge b\left(J H^{*}\right)\right)+2 X_{+} \wedge\left(b\left(H^{*}\right) \wedge H x+b\left(J H^{*}\right) \wedge J H x+\right. \\
\left.+b\left(X_{+}^{*}\right) \wedge X_{+} x+b\left(J X_{+}^{*}\right) \wedge J X_{+} x+b\left(X_{-}^{*}\right) \wedge X_{-} x+b\left(J X_{-}^{*}\right) \wedge J X_{-} x\right) .
\end{gathered}
$$

Now we substitute previously computed solutions, neglecting terms which do not contribute to the factor at $e_{-} \wedge e_{1}, e_{-} \wedge e_{2}$. We have (apart from $2 X_{+}$)

$\left(x^{-} e_{1}-y^{-} e_{2}\right) \wedge x+y \wedge\left(-y^{-} e_{1}-x^{-} e_{2}\right)+\left(x^{-} e_{1}-y^{-} e_{2}\right) \wedge\left(-x^{-} e_{-}\right)+x \wedge 2 x^{-} e_{1}+y \wedge\left(-2 x^{-} e_{2}\right)$.

It is easy to write the part of $[b, b]$, proportional to $2 X_{+} \wedge e_{-} \wedge e_{1}$ :

$$
2 X_{+} \wedge e_{-} \wedge e_{1} \cdot\left(-x^{-2}-y^{-2}+x^{-2}+2 x^{-2}\right)=2 X_{+} \wedge e_{-} \wedge e_{1} \cdot\left(2 x^{-2}-y^{-2}\right)
$$

and to $2 X_{+} \wedge e_{-} \wedge e_{2}$ :

$$
2 X_{+} \wedge e_{-} \wedge e_{2} \cdot\left(x^{-} y^{-}-y^{-} x^{-}-x^{-} y^{-}-2 x^{-} y^{-}\right)=2 X_{+} \wedge e_{-} \wedge e_{2} \cdot\left(-3 x^{-} y^{-}\right)
$$


Similar calculation shows that the term proportional to $2 J X_{+} \wedge e_{2} \wedge e_{-}$is

$$
2 J X_{+} \wedge e_{2} \wedge e_{-} \cdot\left(2 y^{-2}-x^{-2}\right) .
$$

Looking at (70), we see that

$$
2 x^{-2}-y^{-2}=2 y^{-2}-x^{-2}, \quad x^{-} y^{-}=0,
$$

which means that $x^{-}=0=y^{-}$and $t=0$. In particular, $b\left(X_{-}^{*}\right)=0=b\left(J X_{-}^{*}\right)$ and

$$
b=X_{+} \wedge\left(x^{+} e_{+}+x^{1} e_{1}+x^{2} e_{2}\right)+J X_{+} \wedge\left(y^{+} e_{+}+y^{1} e_{1}+y^{2} e_{2}\right)+H \wedge \frac{1}{2}\left(x^{1}-y^{2}\right) e_{+}+J H \wedge z^{+} e_{+} .
$$

We shall simplify this general form, using appropriate automorphisms of $\mathfrak{g}$. First, note that

$$
(-v) c=J X_{+} v \wedge X_{+}+J X_{+} \wedge X_{+} v=X_{+} \wedge\left(2 v^{-} e_{2}+v^{2} e_{+}\right)+J X_{+} \wedge\left(2 v^{-} e_{1}+v^{1} e_{+}\right),
$$

hence transforming $b$ into $b+(-v) c\left(\right.$ as in (55)) we may assume that $x^{+}=0=y^{+}$and $x^{2}+y^{1}=0$ in $(75)$. Secondly, the one-parameter group of internal automorphisms generated by $J H$ leaves $c$ invariant and transforms $b$ according to $\dot{b}=J H \cdot b$, i.e.

$$
\dot{x}^{1}=x^{2}-y^{1}=\dot{y}^{2}, \quad \dot{x}^{2}=-\left(x^{1}+y^{2}\right)=-\dot{y}^{1},
$$

or

$$
\left(x^{1}-y^{2}\right)^{\cdot}=2\left(x^{2}+y^{1}\right), \quad\left(x^{2}-y^{1}\right)^{\cdot}=-2\left(x^{1}+y^{2}\right)
$$

and $\left(x^{1}+y^{2}\right)^{\bullet}=0=\left(x^{2}-y^{1}\right)^{\bullet}$, hence one can afford $x^{2}-y^{1}=0$. This implies $x^{2}=0=y^{1}$ (we already had $x^{2}+y^{1}=0$ ) and we have the following simplified form of $b$ :

$$
b=x^{1} X_{+} \wedge e_{1}+y^{2} J X_{+} \wedge e_{2}+\frac{1}{2}\left(x^{1}-y^{2}\right) H \wedge e_{+}+z^{+} J H \wedge e_{+} \cdot
$$

Now we can finally solve (40). We have

$$
[b, b]=\left(x^{1}+y^{2}\right)\left(J X_{+} \wedge e_{+} \wedge\left(y^{2} e_{2}+2 z^{+} e_{1}\right)-X_{+} \wedge e_{+} \wedge\left(x^{1} e_{1}+2 z^{+} e_{2}\right)\right) .
$$

For $a=e_{+} \wedge\left(\alpha_{1} e_{1}+\alpha_{2} e_{2}\right)+e_{-} \wedge\left(\beta_{1} e_{1}+\beta_{2} e_{2}\right)+\gamma e_{-} \wedge e_{+}+\delta e_{1} \wedge e_{2}$ we have also (see (71))

$$
\begin{gathered}
{[c, a]=J X_{+} \wedge\left(\beta_{1} e_{-} \wedge e_{+}+2 \beta_{2} e_{1} \wedge e_{2}-2 \gamma e_{+} \wedge e_{1}+\delta e_{+} \wedge e_{2}\right)+} \\
-X_{+} \wedge\left(2 \beta_{1} e_{1} \wedge e_{2}-\beta_{2} e_{-} \wedge e_{+}+2 \gamma e_{+} \wedge e_{2}+\delta e_{+} \wedge e_{1}\right) .
\end{gathered}
$$

It follows that $2[c, a]+[b, b]=0$ if and only if $\beta_{1}=\beta_{2}=\gamma=0$ and

$$
z^{+}\left(x^{1}+y^{2}\right)=0, \quad-2 \delta=y^{2}\left(x^{1}+y^{2}\right)=x^{1}\left(x^{1}+y^{2}\right) .
$$

There are two possibilities:

1. $x^{1}+y^{2}=0, \delta=0$, i.e.

$$
b=x^{1}\left(X_{+} \wedge e_{1}-J X_{+} \wedge e_{2}+H \wedge e_{+}\right)+z^{+} J H \wedge e_{+}, \quad a=e_{+} \wedge\left(\alpha_{1} e_{1}+\alpha_{2} e_{2}\right),
$$


2. $x^{1}+y^{2} \neq 0, z^{+}=0, x^{1}=y^{2}, \delta=-\left(x^{1}\right)^{2}$, i.e.

$$
b=x^{1}\left(X_{+} \wedge e_{1}+J X_{+} \wedge e_{2}\right), \quad a=e_{+} \wedge\left(\alpha_{1} e_{1}+\alpha_{2} e_{2}\right)-\left(x^{1}\right)^{2} e_{1} \wedge e_{2} .
$$

Of course, $(79)$ is the case 4 in the table. Note, that since $b$ in $(78)$ is $J H$-invariant (and $c$ also is), one can transform $a$ to the following form:

$$
a=\alpha e_{+} \wedge e_{1}
$$

because $J H$ generates rotations in the $e_{1}, e_{2}$-plane. If $z^{+}=0$, we obtain case 3 in the table. If $z^{+} \neq 0$ we can get rid of $a$ in (78) as follows. First we transform the whole $r$ as in (55) with $v=v^{1} e_{1}+v^{2} e_{2}$, which gives new $b($ cf. (76) ) and $a$ :

$$
\begin{aligned}
& b=x^{1}\left(X_{+} \wedge e_{1}-J X_{+} \wedge e_{2}+H \wedge e_{+}\right)+z^{+} J H \wedge e_{+}+X_{+} \wedge v^{2} e_{+}+J X_{+} \wedge v^{1} e_{+} \\
& a=e_{+} \wedge\left(\alpha_{1} e_{1}+\alpha_{2} e_{2}\right)+x^{1}\left(v^{1} e_{+} \wedge e_{1}+v^{2} e_{+} \wedge e_{2}\right)+z^{+}\left(v^{2} e_{1}-v^{1} e_{2}\right) \wedge e_{+} .
\end{aligned}
$$

We choose $v^{1}, v^{2}$ such that $a=0$. Now observe that $X_{+} c=0, J X_{+} c=0, X_{+} b=-z^{+} J X_{+} \wedge$ $e_{+}$and $J X_{+} b=z^{+} X_{+} \wedge e_{+}$, hence the automorphism groups generated by $X_{+}$and $J X_{+}$ change only $v^{1}$ and $v^{2}$, respectively, according to

$$
\frac{d}{d t} v^{1}=-2 z^{+}, \quad \frac{d}{d s} v^{2}=2 z^{+}
$$

(parameters $t$ and $s$ correspond, respectively, to $X_{+}$and $J X_{+}$). Using these transformations we can afford $v^{1}=0=v^{2}$ (due to $z^{+} \neq 0$ ), which is the case 2 in the table.

\section{$4.3 c=H \wedge X_{+}-J H \wedge J X_{+}+\gamma J X_{+} \wedge X_{+}$}

Calculation of brackets (43) and corresponding cocycle condition (42) gives

$$
\begin{array}{rlrl}
{\left[H^{*}, X_{-}^{*}\right]} & =0 & 0 & =X_{+} b\left(X_{-}^{*}\right) \\
{\left[H^{*}, J X_{-}^{*}\right]=0} & 0 & =X_{+} b\left(J X_{-}^{*}\right) \\
{\left[J H^{*}, X_{-}^{*}\right]} & =0 & 0 & =J X_{+} b\left(X_{-}^{*}\right) \\
{\left[J H^{*}, J X_{-}^{*}\right]} & =0 & 0 & =J X_{+} b\left(J X_{-}^{*}\right) \\
{\left[H^{*}, J H^{*}\right]} & =0 & 0 & =X_{+} b\left(J H^{*}\right)+J X_{+} b\left(H^{*}\right) \\
{\left[X_{+}^{*}, X_{-}^{*}\right]} & =-X_{-}^{*} & b\left(X_{-}^{*}\right) & =\left(H+\gamma J X_{+}\right) b\left(X_{-}^{*}\right) \\
{\left[X_{+}^{*}, J X_{-}^{*}\right]=} & -J X_{-}^{*} & b\left(J X_{-}^{*}\right) & =\left(H+\gamma J X_{+}\right) b\left(J X_{-}^{*}\right) \\
{\left[J X_{+}^{*}, X_{-}^{*}\right]=-J X_{-}^{*}} & -b\left(J X_{-}^{*}\right) & =\left(J H+\gamma J X_{+}\right) b\left(X_{-}^{*}\right) \\
{\left[J X_{+}^{*}, J X_{-}^{*}\right]=X_{-}^{*}} & b\left(X_{-}^{*}\right) & =\left(J H+\gamma J X_{+}\right) b\left(J X_{-}^{*}\right) \\
{\left[H^{*}, X_{+}^{*}\right]=H^{*}+2 \gamma J X_{-}^{*}} & b\left(H^{*}\right)+2 \gamma b\left(J X_{-}^{*}\right)=X_{+} b\left(X_{+}^{*}\right)+\left(H+\gamma J X_{+}\right) b\left(H^{*}\right) \\
{\left[J H^{*}, X_{+}^{*}\right]=J H^{*}-2 \gamma X_{-}^{*}} & b\left(J H^{*}\right)-2 \gamma b\left(X_{-}^{*}\right)=-J X_{+} b\left(X_{+}^{*}\right)+\left(H+\gamma J X_{+}\right) b\left(J H^{*}\right) \\
{\left[H^{*}, J X_{+}^{*}\right]=J H^{*}+2 \gamma X_{-}^{*}} & b\left(J H^{*}\right)+2 \gamma b\left(X_{-}^{*}\right)=X_{+} b\left(J X_{+}^{*}\right)-\left(J H+\gamma X_{+}\right) b\left(H^{*}\right) \\
{\left[J X_{+}^{*}, J H^{*}\right]=H^{*}-2 \gamma J X_{-}^{*}} & b\left(H^{*}\right)-2 \gamma b\left(J X_{-}^{*}\right)=J X_{+} b\left(J X_{+}^{*}\right)+\left(J H+\gamma X_{+}\right) b\left(J H^{*}\right) \\
{\left[X_{+}^{*}, J X_{+}^{*}\right]=-2 \gamma H^{*}} & 2 \gamma b\left(H^{*}\right)=\left(J H+\gamma X_{+}\right) b\left(X_{+}^{*}\right)+\left(H+\gamma J X_{+}\right) b\left(J X_{+}^{*}\right)
\end{array}
$$


(and $\left[X_{-}^{*}, J X_{-}^{*}\right]=0$ ). We have suppressed the subscript ' $c$ ' in the bracket. The first four equations imply that equations from the sixth to the ninth take the form

$$
\begin{aligned}
b\left(X_{-}^{*}\right) & =H b\left(X_{-}^{*}\right) \\
b\left(J X_{-}^{*}\right) & =H b\left(J X_{-}^{*}\right) \\
-b\left(J X_{-}^{*}\right) & =J H b\left(X_{-}^{*}\right) \\
b\left(X_{-}^{*}\right) & =J H b\left(J X_{-}^{*}\right) .
\end{aligned}
$$

First two of the above equations imply that $b\left(X_{-}^{*}\right)$ and $b\left(J X_{-}^{*}\right)$ are proportional to $e_{+}$and then the last two equations imply $b\left(X_{-}^{*}\right)=0=b\left(J X_{-}^{*}\right)$. What remains is the following set of equations:

$$
\begin{aligned}
X_{+} b\left(J H^{*}\right) & =-J X_{+} b\left(H^{*}\right) \\
b\left(H^{*}\right) & =X_{+} b\left(X_{+}^{*}\right)+\left(H+\gamma J X_{+}\right) b\left(H^{*}\right) \\
b\left(J H^{*}\right) & =-J X_{+} b\left(X_{+}^{*}\right)+\left(H+\gamma J X_{+}\right) b\left(J H^{*}\right) \\
b\left(J H^{*}\right) & =X_{+} b\left(J X_{+}^{*}\right)-\left(J H+\gamma X_{+}\right) b\left(H^{*}\right) \\
b\left(H^{*}\right) & =J X_{+} b\left(J X_{+}^{*}\right)+\left(J H+\gamma X_{+}\right) b\left(J H^{*}\right) \\
2 \gamma b\left(H^{*}\right) & =\left(J H+\gamma X_{+}\right) b\left(X_{+}^{*}\right)+\left(H+\gamma J X_{+}\right) b\left(J X_{+}^{*}\right) .
\end{aligned}
$$

Knowing that $\operatorname{ker} X_{+}=\left\langle e_{+}, e_{2}\right\rangle, X_{+} e_{1}=e_{+}, X_{+} e_{-}=2 e_{1}$, ker $J X_{+}=\left\langle e_{+}, e_{1}\right\rangle, J X_{+} e_{2}=$ $-e_{+}, J X_{+} e_{-}=-2 e_{2}$, one can easily solve (82):

$$
b\left(H^{*}\right)=\alpha e_{+}+\beta e_{1}+\lambda e_{2}, \quad b\left(J H^{*}\right)=\mu e_{+}+\lambda e_{1}+\rho e_{2}, \quad \alpha, \beta, \lambda, \mu, \rho \in \mathbb{R} .
$$

Setting $b\left(X_{+}^{*}\right)=: x$, we can write 83 as follows:

$$
\alpha e_{+}+\beta e_{1}+\lambda e_{2}=x^{1} e_{+} 2 x^{-} e_{1}+\alpha e_{+}-\lambda e_{+} .
$$

It means that $\lambda=0=x^{1}, \beta=2 x^{-}$. From (84) we get

$$
\mu e_{+}+\rho e_{2}=x^{2} e_{+}+2 x^{-} e_{2}+\mu e_{+}-\gamma \rho e_{+},
$$

hence $\rho=2 x^{-}, x^{2}=\gamma \rho=2 \gamma x^{-}$. Recall that we have now

$$
b\left(H^{*}\right)=\alpha e_{+}+2 x^{-} e_{1}, \quad b\left(J H^{*}\right)=\mu e_{+}+2 x^{-} e_{2} .
$$

Setting $b\left(J X_{+}^{*}\right)=: y$, we get from $(85)$

$$
\mu e_{+} 2 x^{-} e_{2}=y^{1} e_{+}+2 y^{-} e_{1}+2 x^{-} e_{2}-2 \gamma x^{-} e_{+},
$$

hence we get $y^{-}=0$ and $y^{1}=\mu+2 \gamma x^{-}$. Equation (86) yields

$$
\alpha e_{+}+2 x^{-} e_{1}=-y^{2} e_{+}+2 x^{-} e_{1},
$$

hence $y^{2}=-\alpha$. Finally, (87) yields

$$
2 \gamma\left(\alpha e_{+}+2 x^{-} e_{1}\right)=x^{2} e_{1}+2 \gamma x^{-} e_{1}+y^{+} e_{+}-\gamma y^{2} e_{+} .
$$


Since $x^{2}=2 \gamma x^{-}, y^{2}=-\alpha$, from this equation we get $y^{+}=\gamma \alpha$. Concluding, the general solution of (42) is

$$
\begin{gathered}
b=H \wedge\left(\alpha e_{+}+2 x^{-} e_{1}\right)+J H \wedge\left(\mu e_{+}+2 x^{-} e_{2}\right)+ \\
+X_{+} \wedge\left(x^{+} e_{+}+x^{-} e_{-}+2 \gamma x^{-} e_{2}\right)+J X_{+} \wedge\left(\gamma \alpha e_{+}+\left(\mu+2 \gamma x^{-}\right) e_{1}-\alpha e_{2}\right) .
\end{gathered}
$$

Comparing this with $(-v) c$ for a general $v \in V$,

$$
\begin{gathered}
(-v) c=H \wedge\left(v^{1} e_{+}+2 v^{-} e_{1}\right)+J H \wedge\left(v^{2} e_{+}+2 v^{-} e_{2}\right)+ \\
+X_{+} \wedge\left(\left(\gamma v^{2}-v^{+}\right) e_{+}+v^{-} e_{-}+2 \gamma v^{-} e_{2}\right)+J X_{+} \wedge\left(\gamma v^{1} e_{+}+\left(v^{2}+2 \gamma v^{-}\right) e_{1}-v^{1} e_{2}\right),
\end{gathered}
$$

it is easy to see that $b=(-v) c$ for $v^{1}=\alpha, v^{2}=\mu, v^{-}=x^{-}, v^{+}=\gamma \mu-x^{+}$. Therefore we can always assume that $b=0$.

Now we shall show that $[c, a]=0 \Longrightarrow a=0$ for $a \in \bigwedge^{2} V$. Indeed,

$$
[c, a]=H \wedge X_{+} a-J H \wedge J X_{+} a-X_{+} \wedge\left(H a+\gamma J X_{+} a\right)+J X_{+} \wedge\left(J H a+\gamma X_{+} a\right)
$$

\begin{tabular}{|c|c|c|c|c|c|}
\hline$\left[H^{*}, X_{+}^{*}\right]$ & $=H^{*}$ & $1^{\bullet}$ & $b\left(H^{*}\right)$ & $=$ & $X_{+} b\left(X_{+}^{*}\right)+H b\left(H^{*}\right)$ \\
\hline$\left[H^{*}, J X_{+}^{*}\right]$ & $=J H^{*}$ & $2^{\bullet}$ & $b\left(J H^{*}\right)$ & $=$ & $X_{+} b\left(J X_{+}^{*}\right)$ \\
\hline$\left[H^{*}, X_{-}^{*}\right]$ & $=0$ & $3^{\bullet}$ & 0 & $=$ & $X_{+} b\left(X_{-}^{*}\right)$ \\
\hline$\left[H^{*}, J X_{-}^{*}\right]$ & $=0$ & $4^{\bullet}$ & 0 & $=$ & $X_{+} b\left(J X_{-}^{*}\right)$ \\
\hline$\left[X_{+}^{*}, J H^{*}\right]$ & $=0$ & $5^{\bullet}$ & 0 & $=$ & $H b\left(J H^{*}\right)$ \\
\hline$\left[H^{*}, J H^{*}\right]$ & $=-2 J X_{-}^{*}$ & $6^{\bullet}$ & $2 b\left(J X_{-}^{*}\right)$ & $=$ & $-X_{+} b\left(J H^{*}\right)$ \\
\hline$\left[X_{+}^{*}, J X_{+}^{*}\right]$ & $=J X_{+}^{*}$ & $7^{\bullet}$ & $b\left(J X_{+}^{*}\right)$ & $=$ & $-H b\left(J X_{+}^{*}\right)$ \\
\hline$\left[X_{+}^{*}, J X_{-}^{*}\right]$ & $=-J X_{-}^{*}$ & $8^{\bullet}$ & $b\left(J X_{-}^{*}\right)$ & $=$ & $H b\left(J X_{-}^{*}\right)$ \\
\hline$\left[X_{+}^{*}, X_{-}^{*}\right]$ & $=-X_{-}^{*}$ & $9^{\bullet}$ & $b\left(X_{-}^{*}\right)$ & $=$ & $H b\left(X_{-}^{*}\right)$. \\
\hline
\end{tabular}

is zero if and only if $X_{+} a=0, J X_{+} a=0, H a=0$ and $J H a=0$. But the commutant of $\left\{H, X_{+}\right\}$in $\mathfrak{h}$ is zero.

\section{$4.4 c=H \wedge X_{+}$}

We calculate brackets (43) relevant for the cocycle condition (42):

It follows from $7^{\bullet}-9^{\bullet}$ that $b\left(J X_{+}^{*}\right)=\alpha e_{-}, b\left(J X_{-}^{*}\right)=\beta e_{+}, b\left(X_{-}^{*}\right)=\gamma e_{+}$and this implies $3^{\bullet}-4^{\bullet}$. From $2^{\bullet}$ we obtain $b\left(J H^{*}\right)=2 \alpha e_{1}$ which implies $5^{\bullet}$. $6^{\bullet}$ means $2 \beta e_{+}=-X_{+}\left(2 \alpha e_{1}\right)=$ $-2 \alpha e_{+}$, hence $\beta=-\alpha$. The only remaining condition is $1^{\bullet}$ :

$$
(1-H) b\left(H^{*}\right)=X_{+} b\left(X_{+}^{*}\right)
$$

Denoting $b\left(X_{+}^{*}\right)=: x, b\left(H^{*}\right)=: y$ we obtain

$$
2 y^{-} e_{-}+y^{1} e_{1}+y^{2} e_{2}=x^{1} e_{+}+2 x^{-} e_{1}
$$

i.e. $x^{1}=y^{-}=y^{2}=0, y^{1}=2 x^{-}$. The general solution of the cocycle condition is therefore $b=H \wedge\left(y^{+} e_{+}+2 x^{-} e_{1}\right)+J H \wedge 2 \alpha e_{1}+X_{+} \wedge\left(x^{+} e_{+}+x^{-} e_{-}+x^{2} e_{2}\right)+\alpha J X_{+} \wedge e_{-}+\gamma X \wedge e_{+}-\alpha J X \wedge e_{+}$. 
Adding to this

$$
(-v) c=H \wedge X_{+} v-X_{+} \wedge H v=H \wedge\left(v^{1} e_{+}+2 v^{-} e_{1}\right)-X_{+} \wedge\left(v^{+} e_{+}-v^{-} e_{-}\right)
$$

for a suitable $v \in V$, we get a simpler form of $b$ :

$$
b=2 \alpha J H \wedge e_{1}+x^{2} X_{+} \wedge e_{2}+\alpha J X_{+} \wedge e_{-}+\gamma X_{-} \wedge e_{+}-\alpha J X_{-} \wedge e_{+} .
$$

We have $b=\alpha b_{0}+\beta b_{1}+\gamma b_{2}$, where $\alpha, \beta, \gamma$ are some constants and

$$
\begin{aligned}
b_{0} & =2 J H \wedge e_{1}+J X_{+} \wedge e_{-}-J X_{-} \wedge e_{+} \\
b_{1} & =X_{+} \wedge e_{2} \\
b_{2} & =X_{-} \wedge e_{+}
\end{aligned}
$$

It is easy to see that $b_{0}=2 b_{e_{2}}$ (formula (44)) and $X_{+} e_{2}=0$, hence $\left[b_{0}, b_{0}\right]=4 \Omega$ and

$$
\left[\alpha b_{0}+\beta b_{1}, \alpha b_{0}+\beta b_{1}\right]=\alpha^{2}\left[b_{0}, b_{0}\right]=4 \alpha^{2} \Omega
$$

(cf. (45)). Since

$\left[b_{2}, b_{0}\right]=2 J X_{-} \wedge e_{+} \wedge e_{1}-2 X_{-} \wedge e_{+} \wedge e_{2}, \quad\left[b_{2}, b_{1}\right]=-2 H \wedge e_{+} \wedge e_{2}, \quad\left[b_{2}, b_{2}\right]=4 X_{-} \wedge e_{+} \wedge e_{1}$,

we have

$[b, b]=\alpha^{2}\left[b_{0}, b_{0}\right]+2 \gamma \alpha\left(2 J X_{-} \wedge e_{+} \wedge e_{1}-2 X_{-} \wedge e_{+} \wedge e_{2}\right)-2 \gamma \beta \cdot 2 H \wedge e_{+} \wedge e_{2}+4 \gamma^{2} X_{-} \wedge e_{+} \wedge e_{1}$

The element

$$
2[c, a]+\left([b, b]-\alpha^{2}\left[b_{0}, b_{0}\right]\right)
$$

is proportional to $\Omega$ and has rank at most 4 (there are no terms involving $J H$ and $J X_{+}$), hence it is zero. In particular (taking the term with $X_{-} \wedge e_{+} \wedge e_{1}$ ) we have $\gamma=0$. Finally we have

$$
b=\alpha b_{0}+\beta b_{1}
$$

and $[c, a]=0$. It follows that $H a=0=X_{+} a$, hence $a=0$ (cf. the end of the previous section). This is the item 6 of the table.

\section{The proof for $c=0$}

We consider the case when $t=0$, hence equations (38)-(41) for $r=a+b$ reduce to

$$
[b, b]=0, \quad[b, a]=0 .
$$

Since $b$ is a triangular $r$-matrix,

$$
b\left(\mathfrak{g}^{*}\right)=V_{0}+\mathfrak{h}_{0},
$$

where $\mathfrak{h}_{0}:=b\left(V^{*}\right) \subset \mathfrak{h}, V_{0}:=b\left(\mathfrak{h}^{*}\right) \subset V$, is a Lie subalgebra of $\mathfrak{g}=V \rtimes \mathfrak{h}$. It follows that $\mathfrak{h}_{0}$ is a Lie subalgebra of $\mathfrak{h}$ and $\left[\mathfrak{h}_{0}, V_{0}\right] \subset V_{0}$, therefore $b\left(\mathfrak{g}^{*}\right)=V_{0} \rtimes \mathfrak{h}_{0}$. Of course, $b$ is a 
triangular $r$-matrix on the smaller Lie algebra $V_{0} \rtimes \mathfrak{h}_{0}$. Let $b(\cdot)$ denote the linear bijection from $V_{0}^{*}$ to $\mathfrak{h}_{0}$ defined by $b$. Equation $[b, b]=0$ is equivalent to

$$
[b(\xi), b(\eta)]=b\left([\xi, \eta]_{b}\right), \quad \xi, \eta \in V_{0}^{*}
$$

(cf. (11)). Applying the inverse map $f: \mathfrak{h}_{0} \rightarrow V_{0}^{*}$ of $b(\cdot)$ to the above equation changes it from quadratic to a linear (!) one:

$$
f([X, Y])=X f(Y)-Y f(X), \quad X, Y \in \mathfrak{h}_{0},
$$

which says that $f$ is just a cocycle (on $\mathfrak{h}_{0}$ with values in $V_{0}^{*}$ ).

We consider four possible cases of $\operatorname{dim} V_{0}=\operatorname{dim} \mathfrak{h}_{0}$ separately.

\section{$5.1 \operatorname{dim} V_{0}=4$}

We shall show that there are no solutions of this type. The following lemma is not difficult.

Lemma 5.1 Any four-dimensional Lie subalgebra $\mathfrak{h}_{0}$ of $\mathfrak{h}=s l(2, \mathbb{C})$ can be transformed by an internal automorphism to

$$
\left\{\left(\begin{array}{rr}
z & w \\
0 & -z
\end{array}\right): z, w \in \mathbb{C}\right\}=\left\langle H, J H, X_{+}, J X_{+}\right\rangle .
$$

Assuming that $\mathfrak{h}_{0}$ is given by (90), we are looking for cocycles $f: \mathfrak{h}_{0} \rightarrow V^{*}$. We can replace $V^{*}$ by the isomorphic h-module $V$. Set $f(H)=: h, f(J H)=: k, f\left(X_{+}\right)=: x$ and $f\left(J X_{+}\right)=: y$. The map $f$ is a cocycle if and only if vectors $h, k, x, y$ satisfy

$$
\begin{aligned}
H k & =J H h \\
X_{+} y & =J X_{+} x \\
x & =H x-X_{+} h \\
y & =H y-J X_{+} h \\
y & =J H x-X_{+} k \\
-x & =J H y-J X_{+} k .
\end{aligned}
$$

The first two equations are equivalent to $h=h^{+} e_{+}+h^{-} e_{-}, k=k^{1} e_{1}+k^{2} e_{2}, x=x^{+} e_{+}+$ $x^{1} e_{1}+x^{2} e_{2}, y=y^{+} e_{+}-x^{2} e_{1}+y^{2} e_{2}$. Inserting this in (91) gives $x^{2}=0, x^{1}=-2 h^{-}$. Inserting in (92) gives $y^{2}=2 h^{-}$. Then the last two equations yield $y^{+}=-k^{1}, x^{+}=k^{2}$. The general solution is therefore as follows:

$$
h=h^{+} e_{+}+h^{-} e_{-}, \quad k=k^{1} e_{1}+k^{2} e_{2}, \quad x=k^{2} e_{+}-2 h^{-} e_{1}, \quad y=-k^{1} e_{+}+2 h^{-} e_{2} .
$$

These vectors are however linearly dependent:

$$
\operatorname{det}\left[\begin{array}{cccc}
h^{-} & 0 & 0 & 0 \\
h^{+} & 0 & k^{2} & -k^{1} \\
0 & k^{1} & -2 h^{-} & 0 \\
0 & k^{2} & 0 & 2 h^{-}
\end{array}\right]=0,
$$

hence $f$ cannot be a bijection (this ends the proof). 


\section{$5.2 \operatorname{dim} V_{0}=3$}

There are three types of 3-dimensional subspaces $V_{0}$ of $V$ :

1. space-like: $\left.g\right|_{V_{0}}$ has signature $(0,3)$. Then $\mathfrak{h}_{0} \cong o(0,3)$.

2. 3D-Minkowski : $\left.g\right|_{V_{0}}$ has signature $(1,2)$. Then $\mathfrak{h}_{0} \cong o(1,2)$.

3. tangent to the light cone: $\left.g\right|_{V_{0}}$ has signature $(0,2)$.

In the first two cases $\mathfrak{h}_{0}$ is simple and $f$ has to be a coboundary:

$$
\left.f(X)=X \xi, \quad X \in \mathfrak{h}_{0} \quad \text { (for some } \xi \in V_{0}^{*}\right) .
$$

Since each $\xi \in V^{*}$ has a nontrivial isotropy, $f$ cannot be bijective.

In the third case we can assume the standard form $V_{0}=\left\langle e_{+}, e_{1}, e_{2}\right\rangle$. We have

$$
\mathfrak{h}_{0} \subset\left\langle H, J H, X_{+}, J X_{+}\right\rangle
$$

because $\mathfrak{h}_{0}$ is contained in the subalgebra stabilizing $V_{0}$.

Lemma $5.2 \quad \mathfrak{h}_{0} \supset\left\langle X_{+}, J X_{+}\right\rangle$.

Proof: We set $\mathfrak{n}:=\left\langle X_{+}, J X_{+}\right\rangle$. Since $\operatorname{dim} \mathfrak{h}_{0}=3$ and $\operatorname{dim} \mathfrak{n}=2$, there exists $0 \neq Y \in$ $\mathfrak{h}_{0} \cap \mathfrak{n}$. If $\mathfrak{h}_{0}$ does not contain $\mathfrak{n}$, then $\mathfrak{h}_{0}+\mathfrak{n}=\left\langle X_{+}, J X_{+}, \lambda H+\mu J H\right\rangle$, hence $J H \in \mathfrak{h}_{0}+\mathfrak{n}$ and therefore

$$
J Y=[J H, Y] \in \mathfrak{h}_{0}
$$

i.e. $\mathfrak{n}=\langle Y, J Y\rangle \subset \mathfrak{h}_{0}$.

From the above lemma it follows that

$$
\mathfrak{h}_{0}=\left\langle X_{+}, J X_{+}, \lambda H+\mu J H\right\rangle
$$

where $\lambda^{2}+\mu^{2} \neq 0$. Let $\left(e^{+}, e^{1}, e^{2}\right)$ be the basis in $V_{0}^{*}$ dual to $\left(e_{+}, e_{1}, e_{2}\right)$. The coordinates of an element $x \in V_{0}^{*}$ in this basis are denoted by $x_{+}, x_{1}, x_{2}$. We calculate also the action of $\mathfrak{h}_{0}$ on $V_{0}^{*}$ :

$$
\begin{aligned}
& X_{+} e^{+}=-e^{1}, \quad J X_{+} e^{+}=e^{2}, \quad(\lambda H+\mu J H) e^{+}=-\lambda e^{+}, \\
& X_{+} e^{1}=0, \quad J X_{+} e^{1}=0, \quad(\lambda H+\mu J H) e^{1}=-\mu e^{2}, \\
& X_{+} e^{2}=0, \quad J X_{+} e^{2}=0, \quad(\lambda H+\mu J H) e^{2}=\mu e^{1} \text {. }
\end{aligned}
$$

Let $f: \mathfrak{h}_{0} \rightarrow V_{0}^{*}$ be a linear map and $f\left(X_{+}\right)=: x, f\left(J X_{+}\right)=: y, f(\lambda H+\mu J H)=: z$. It is a cocycle if and only if

$$
\begin{aligned}
X_{+} y & =J X_{+} x \\
(\lambda H+\mu J H) x-X_{+} z & =\lambda x+\mu y \\
(\lambda H+\mu J H) y-J X_{+} z & =-\mu x+\lambda y .
\end{aligned}
$$


The first equation is equivalent to $x_{+}=0=y_{+}$. Since $H x=0=H y$ and $X_{+} z=-z_{+} e^{1}$, $J X_{+} z=z_{+} e^{2}$, equations (94)-(95) are equivalent to

$$
\mu J H w+z_{+}\left(e^{1}-i e^{2}\right)=(\lambda-i \mu) w,
$$

where $w:=x+i y$ (just add (95) multiplied by $i$ to (94)), or to

$$
\mu(J H+i) w+z_{+}\left(e^{1}-i e^{2}\right)=\lambda w .
$$

Since $J H\left(e^{1}-i e^{2}\right)=-i\left(e^{1}-i e^{2}\right)$, (96) is the decomposition of $\lambda w$ on components belonging to eigenspaces of $J H$ (we know that $(J H)^{2}=-1$ on the subspace spanned by $e^{1}, e^{2}$ ). If $\lambda=0$ then $z_{+}=0$ and $x, y, z$ are linearly dependent. In order $f$ to be bijective we must have therefore $\lambda \neq 0$. In such a case we can assume in (93) and in the sequel that $\lambda=1$ :

$$
\mu(J H+i) w+z_{+}\left(e^{1}-i e^{2}\right)=w .
$$

Substituting here $w=w_{+i}+w_{-i}$, where $w_{+i}$ and $w_{-i}$ are the eigenvectors of $J H$ corresponding to $+i$ and $-i$, respectively, we obtain $w_{+i}=0$. Therefore we have

$$
w=w_{-i}=z_{+}\left(e^{1}-i e^{2}\right)
$$

hence

$$
x=z_{+} e^{1}, \quad y=-z_{+} e^{2}, \quad z=z_{+} e^{+}+z_{1} e^{1}+z_{2} e^{2} .
$$

Using the possibility of scaling $b$ (or $f$ ) by a non-zero factor, we can assume that $z_{+}=1$ :

$$
x=e^{1}, \quad y=-e^{2}, \quad z=e^{+}+z_{1} e^{1}+z_{2} e^{2} .
$$

Solving

$$
b_{0}\left(e^{1}\right)=X_{+}, \quad b_{0}\left(-e^{2}\right)=J X_{+}, \quad b_{0}\left(e^{+}+z_{1} e^{1}+z_{2} e^{2}\right)=H+\mu J H,
$$

we obtain

$$
b_{0}\left(e^{1}\right)=X_{+}, \quad b_{0}\left(-e^{2}\right)=J X_{+}, \quad b_{0}\left(e^{+}\right)=H+\mu J H-z_{1} X_{+}+z_{2} J X_{+},
$$

hence finally

$$
b=e_{1} \wedge X_{+}-e_{2} \wedge J X_{+}+e_{+} \wedge\left(H+\mu J H-z_{1} X_{+}+z_{2} J X_{+}\right) .
$$

Now note that

$$
b=b_{e_{+}}+e_{+} \wedge\left(\mu J H-z_{1} X_{+}+z_{2} J X_{+}\right) .
$$

Since $J H, X_{+}, J X_{+}$belong to the isotropy subalgebra of $e_{+}$, the above $b$ is of the form (45) and we can check directly that $[b, b]=0$ (we know it already by the construction):

$$
[b, b]=\left[b_{e_{+}}, b_{e_{+}}\right]=-g\left(e_{+}, e_{+}\right)=0
$$

We have two cases, depending on $\mu$ : 
1. $\mu \neq 0$. In this case one can get rid of $z_{1}, z_{2}$, using the automorphisms generated by $X_{+}, J X_{+}$, since

$$
X_{+} b=e_{+} \wedge \mu\left(-J X_{+}\right), \quad J X_{+} b=e_{+} \wedge \mu X_{+} .
$$

We have then $b=b_{e_{+}}+\mu e_{+} \wedge J H$. Since $J H$ has only imaginary eigenvalues, by Prop. 2.5, adding $a$ does not lead to new solutions, hence we get item 7 of the table.

2. $\mu=0$. The one-parameter group of automorphisms generated by $J H$ acts on $b$ according to the linear system of differential equations $\dot{z}_{1}=z_{2}, \dot{z}_{2}=-z_{1}$. Therefore we can assume that $z_{2}=0: b=b_{e_{+}}+z e_{+} \wedge X_{+}$. Again, there is no need to consider nontrivial $a$, since $X_{+}$is nilpotent. We get then item 8 of the table.

\section{$5.3 \operatorname{dim} V_{0}=2$}

There are three normal forms of a 2-dimensional subspace $V_{0}$ of $V$ :

1. $V_{0}=\left\langle e_{1}, e_{2}\right\rangle$ (space-like : $\left.g\right|_{V_{0}}$ has signature $\left.(0,2)\right)$. Then $\mathfrak{h}_{0}=\langle H, J H\rangle$.

2. $V_{0}=\left\langle e_{+}, e_{-}\right\rangle$(2D-Minkowski : $\left.g\right|_{V_{0}}$ has signature $\left.(1,1)\right)$. Then $\mathfrak{h}_{0}=\langle H, J H\rangle$.

3. $V_{0}=\left\langle e_{1}, e_{+}\right\rangle$(tangent to the light cone : $\left.g\right|_{V_{0}}$ has signature $(0,1)$ ). Then $\mathfrak{h}_{0} \subset$ $\left\langle H, X_{+}, J X_{+}\right\rangle$.

(The simplest way to prove it is to note that 2-dimensional subspaces of $V$ correspond to simple bivectors, i.e. some elements of $\mathfrak{h}$; the classification of the latter is easy.)

In the first case, $b=x \wedge H+y \wedge J H$, where $x, y \in\left\langle e_{1}, e_{2}\right\rangle$. We have

$$
\frac{1}{2}[b, b]=y \wedge J H y \wedge J H+y \wedge J H x \wedge H,
$$

hence $[b, b]=0$ implies the linear dependence of $y, J H y$, i.e. $y=0$. This is in contradiction with $\operatorname{dim} V_{0}=2$.

In the second case, $b=x \wedge H+y \wedge J H$, where $x, y \in\left\langle e_{+}, e_{-}\right\rangle$. We have

$$
\frac{1}{2}[b, b]=x \wedge H x \wedge H+x \wedge H y \wedge J H,
$$

hence $[b, b]=0$ implies $x \wedge H x=0=x \wedge H y$. Since we consider only nonzero $x, y$, this means that there exist $\lambda, \mu$ such that $x=\lambda H x$ and $x=\mu H y$. We have therefore $x=\lambda \mu H^{2} y=\lambda \mu y$. This is in contradiction with $\operatorname{dim} V_{0}=2$.

In the third case, $b$ is of the following form:

$$
b=x \wedge X_{+}+y \wedge J X_{+}+z \wedge H,
$$

where $x=x^{+} e_{+}+x^{1} e_{1}$, etc. A simple calculation shows that $[b, b]=0$ if and only if

$$
\begin{aligned}
& x^{1}\left(x^{1}-z^{+}\right)+2 x^{+} z^{1}=0 \\
& y^{1}\left(x^{1}-z^{+}\right)+2 y^{+} z^{1}=0 \\
& z^{1}\left(x^{1}-z^{+}\right)+2 z^{+} z^{1}=0 .
\end{aligned}
$$


Note that if

$$
\left[\begin{array}{c}
x^{1}-z^{+} \\
2 z^{1}
\end{array}\right]
$$

is a non-zero vector, then $x, y, z$ are in the same one-dimensional subspace. This would mean that $\operatorname{dim} V_{0} \leq 1$. We conclude that $x^{1}-z^{+}=0=z^{1}$ and

$$
b=e_{1} \wedge\left(x^{1} X_{+}+y^{1} J X_{+}\right)+e_{+} \wedge\left(x^{+} X_{+}+y^{+} J X_{+}+x^{1} H\right) .
$$

Now we shall reduce the number of parameters, acting by suitable automorphisms. We consider separately two cases.

Case 1. $x^{1} \neq 0$.

Since $J X_{+} b=-x^{1} e_{+} \wedge J X_{+}$(which means $\dot{y}^{+}=-x^{1}=$ const) and $x^{1} \neq 0$, we can pass to the situation when $y^{+}=0$. Using another group of automorphisms, the one generated by $H$, we get the change of parameters as follows

$$
\dot{x}^{1}=x^{1}, \quad \dot{y}^{1}=y^{1}, \quad \dot{x}^{+}=2 x^{+} .
$$

Using this and the possibility of multiplying $b$ by a nonzero number, we get

$$
b=e_{1} \wedge\left(X_{+}+y^{1} J X_{+}\right)+e_{+} \wedge\left(H+x^{+} X_{+}\right),
$$

where $x^{+}=0, \pm 1$. For $v \in V$ we have

$$
(-v) b=\left(v^{1}-y^{1} v^{2}-2 x^{+} v^{-}\right) e_{1} \wedge e_{+}-2 y^{1} v^{-} e_{1} \wedge e_{2}+v^{-} e_{-} \wedge e_{+},
$$

hence we can assume that $a$ is of the form

$$
a=\alpha e_{+} \wedge e_{2}+e_{-} \wedge\left(\gamma_{1} e_{1}+\gamma_{2} e_{2}\right)+\mu e_{1} \wedge e_{2}
$$

(no component with $e_{-} \wedge e_{+}, e_{1} \wedge e_{+}$). A simple calculation yields

$$
[a, b]=\left(2 \gamma_{1}-y^{1} \gamma_{2}\right) e_{1} \wedge e_{-} \wedge e_{+}+\left(\mu-2 x^{+} \gamma_{2}\right) e_{1} \wedge e_{+} \wedge e_{2}-\gamma_{2} e_{+} \wedge e_{-} \wedge e_{2} .
$$

It follows that $[a, b]=0$ if and only if $a=\alpha e_{+} \wedge e_{2}$, which is item 9 of the table.

Case 2. $x^{1}=0$.

In this case we have

$$
b=y^{1} e_{1} \wedge J X_{+}+e_{+} \wedge\left(x^{+} X_{+}+y^{+} J X_{+}\right)
$$

$y^{1} \neq 0 \neq x^{+}$(because $\left.\operatorname{dim} V_{0}=2\right)$. Since $X_{+} b=-y^{1} e_{+} \wedge J X_{+}$(which means $\dot{y}^{+}=-y^{1}=$ const) and $y^{1} \neq 0$, we can pass to the situation when $y^{+}=0$. Using another group of automorphisms, the one generated by $H$, we get the change of parameters as follows

$$
\dot{y}^{1}=y^{1}, \quad \dot{x}^{+}=2 x^{+} .
$$

Using this and the possibility of multiplying $b$ by a nonzero number, we get

$$
b= \pm e_{1} \wedge J X_{+}+e_{+} \wedge X_{+} .
$$


Now, observe that the reflection $e_{2} \mapsto-e_{2}$ (other elements of the basis unchanged) yields an automorphism of $\mathfrak{g}$ which on $\mathfrak{h}$ coincides with the 'complex conjugation' (if the real part is spanned by $H, X_{+}, X_{-}$), in particular $J X_{+} \mapsto-J X_{+}$. It means that we can choose plus sign in (103):

$$
b=e_{1} \wedge J X_{+}+e_{+} \wedge X_{+}
$$

For $v \in V$ we have

$$
(-v) b=\left(v^{2}-2 v^{-}\right) e_{1} \wedge e_{+}-2 v^{-} e_{1} \wedge e_{2},
$$

hence we can assume that $a$ is of the form

$$
a=e_{-} \wedge\left(\alpha_{1} e_{1}+\alpha_{2} e_{2}\right)+\gamma e_{-} \wedge e_{+}+\alpha e_{+} \wedge e_{2}
$$

(no component with $e_{1} \wedge e_{+}, e_{1} \wedge e_{2}$ ). A simple calculation yields

$$
[a, b]=-\alpha_{2} e_{1} \wedge e_{-} \wedge e_{+}+\left(2 \gamma-2 \alpha_{2}\right) e_{1} \wedge e_{+} \wedge e_{2}
$$

It follows that $[a, b]=0$ if and only if $a=\alpha_{1} e_{-} \wedge e_{1}+\alpha e_{+} \wedge e_{2}$, which is item 10 of the table.

\section{$5.4 \operatorname{dim} V_{0}=1$}

In this case $b=v \wedge X$ for some nonzero $v \in V, X \in \mathfrak{h}$. Since $X$ has to preserve $V_{0}:=\langle v\rangle$, $v$ is an eigenvector of $X$ and $[b, b]=0$ automatically in this case. We can always rescale $X$ in such a way that $X v=0$ or $X v=v$.

The classification procedure is simple. Any nilpotent $X$ is equivalent to $X_{+}$and any semisimple $X$ is equivalent to $\lambda H+\mu J H$. We have then the following possibilities:

\begin{tabular}{c|c}
$X$ & $v$ \\
\hline$X_{+}$ & $v \in\left\langle e_{+}, e_{2}\right\rangle$ \\
$J H$ & $v \in\left\langle e_{+}, e_{-}\right\rangle$ \\
$H$ & $v \in\left\langle e_{1}, e_{2}\right\rangle, v=e_{ \pm}$ \\
$H+\beta J H \quad \beta \neq 0$ & $v=e_{ \pm}$.
\end{tabular}

Note that we can still restrict the possibilities. Namely, we use the automorphisms generated by $J X_{+}, H, J H$ (and scaling) in cases when $X=X_{+}, X=J H, X=H$, respectively, to pass from two-dimensional eigenspaces of $X$ to specific vectors: $e_{+}, e_{2}$ in the first case, $e_{ \pm}, e_{0}, e_{3}$ in the second case and $e_{ \pm}, e_{1}$ in the third. We also use the reflection $e_{3} \mapsto-e_{3}$ in order to replace $e_{-} \wedge J H, e_{-} \wedge H, e_{-} \wedge(H+\beta J H)$ by $e_{+} \wedge J H, e_{+} \wedge H, e_{+} \wedge(H-\beta J H)$, respectively.

The results are presented in the following table, where we have also shown which $a$ satisfy

$$
[a, b]=v \wedge X a=0
$$

how they can be simplified using $(-v) b$ and which still can be simplified using $H$ (in one case also $J H)$ to get the final number of parameters \#. This covers items 11-18 in Table 1 . 


\begin{tabular}{|c|c|c|c|c|}
\hline$b$ & $a$ belongs to & $a+(-v) b$ & still use & $\#$ \\
\hline$e_{2} \wedge X_{+}$ & $\left\langle e_{+} \wedge e_{1}, e_{+} \wedge e_{2}, e_{-} \wedge e_{2}, e_{1} \wedge e_{2}\right\rangle$ & $\left\langle e_{+} \wedge e_{1}, e_{-} \wedge e_{2}\right\rangle$ & $H$ & 1 \\
$e_{+} \wedge X_{+}$ & $\left\langle e_{-} \wedge e_{+}, e_{ \pm} \wedge e_{1}, e_{ \pm} \wedge e_{1}\right\rangle$ & $\left\langle e_{-} \wedge e_{+}, e_{-} \wedge e_{1}, e_{ \pm} \wedge e_{2}\right\rangle$ & $H$ & 3 \\
$e_{0} \wedge J H$ & $\left\langle e_{0} \wedge e_{1}, e_{0} \wedge e_{2}, e_{0} \wedge e_{3}, e_{1} \wedge e_{2}\right\rangle$ & $\left\langle e_{0} \wedge e_{3}, e_{1} \wedge e_{2}\right\rangle$ & & 2 \\
$e_{3} \wedge J H$ & $\left\langle e_{0} \wedge e_{3}, e_{1} \wedge e_{3}, e_{2} \wedge e_{3}, e_{1} \wedge e_{2}\right\rangle$ & $\left\langle e_{0} \wedge e_{3}, e_{1} \wedge e_{2}\right\rangle$ & & 2 \\
$e_{+} \wedge J H$ & $\left\langle e_{+} \wedge e_{1}, e_{+} \wedge e_{2}, e_{+} \wedge e_{3}, e_{1} \wedge e_{2}\right\rangle$ & $\left\langle e_{0} \wedge e_{3}, e_{1} \wedge e_{2}\right\rangle$ & $H$ & 1 \\
$e_{1} \wedge H$ & $\left\langle e_{-} \wedge e_{+}, e_{ \pm} \wedge e_{1}, e_{1} \wedge e_{2}\right\rangle$ & $\left\langle e_{0} \wedge e_{3}, e_{1} \wedge e_{2}\right\rangle$ & & 2 \\
$e_{+} \wedge H$ & $\left\langle e_{+} \wedge e_{1}, e_{+} \wedge e_{2}, e_{+} \wedge e_{3}, e_{1} \wedge e_{2}\right\rangle$ & $\left\langle e_{+} \wedge e_{1}, e_{+} \wedge e_{2}, e_{1} \wedge e_{2}\right\rangle$ & $J H, H$ & 1 \\
$e_{+} \wedge(H+\beta J H)$ & $\left\langle e_{+} \wedge e_{1}, e_{+} \wedge e_{2}, e_{+} \wedge e_{3}, e_{1} \wedge e_{2}\right\rangle$ & $\left\langle e_{1} \wedge e_{2}\right\rangle$ & $H$ & 1 \\
\hline
\end{tabular}

Table 2: The lowest non-zero rank of $b$

\section{$5.5 \quad b_{0}=0$}

The classification of $a \in \bigwedge^{2} V$ is the same as the classification of elements of $\operatorname{sl}(2, \mathbb{C})$. Additionally, we identify proportional elements. The normal forms are $X_{+} \sim e_{1} \wedge e_{+}, J H \sim e_{1} \wedge e_{2}$ and $H+\alpha J H \sim e_{0} \wedge e_{3}+\alpha e_{1} \wedge e_{2}$ (items 19-21).

\section{Final remarks}

1. Unfortunately, we were not able to solve generally the 'classical modified Yang-Baxter equation' $[b, b]=t \Omega, t \neq 0$, in spite of the existence of general solution in the case of simple Lie algebras given by Belavin and Drinfeld [13].

2. According to Remark 1.8 of [2], any solution of (40), (41) with $c=0$ (non-deformed classical Lorentz subgroup) defines directly a quantum Poincaré group. All non-zero solutions with $c=0$ and $t=0$ are given as items $7-21$ of Table 1. Some solutions with $c=0$ and $t \neq 0$ are given in (51), (53), (54).

3. Poisson structures on the Poincaré group acting in 2-dimensional space-time have been classified in [14.

4. The 3-dimensional case is investigated in [15].

5. For each Poisson Poincaré group $G$ there is exactly one Poisson Minkowski space $M$ (with a Poisson action of $G$ on $M$ ), cf. [16, 17].

6. Some classical-mechanical models of particles based on Poisson Poincaré symmetry were discussed in 114, 18, 19]. For a short review see [20].

\section{Acknowledgments}

The author would like to thank to Dr P. Podleś and Dr F. Burstall for valuable discussions.

This research was supported by Polish KBN grant No. 2 P301 02007. 


\section{References}

[1] S. Zakrzewski, Poisson Poincaré groups, in: "Quantum Groups, Formalism and Applications", Proceedings of the XXX Winter School on Theoretical Physics 14-26 February 1994, Karpacz, J. Lukierski, Z. Popowicz, J. Sobczyk (eds.), Polish Scientific Publishers PWN, Warsaw 1995, pp. 433-439 (also: hep-th/9412099).

[2] P. Podleś and S.L. Woronowicz, On the classification of quantum Poincaré groups, hep-th/9412059, UC Berkeley preprint, PAM-632, to appear in Comm. Math. Phys.

[3] W. Greub, S. Halperin and R. Van Stone, Curvature, Connection and Cohomology, Pure and Applied Mathematics, vol. 47, III, Academic Press, New York, 1976.

[4] V. G. Drinfeld, Hamiltonian structures on Lie groups, Lie bialgebras and the meaning of the classical Yang-Baxter equations, Soviet Math. Dokl. 27 (1983), 68-71.

[5] V. G. Drinfeld, Quantum groups, Proc. ICM, Berkeley, 1986, vol.1, 789-820.

[6] M. A. Semenov-Tian-Shansky, Dressing transformations and Poisson Lie group actions, Publ. Res. Inst. Math. Sci., Kyoto University 21 (1985), 1237-1260.

[7] J.-H. Lu and A. Weinstein, Poisson Lie Groups, Dressing Transformations and Bruhat Decompositions, J. Diff. Geom. 31 (1990), 501-526.

[8] N. Bourbaki, Groupes et algèbres de Lie, Chapitre VIII, Hermann 1975.

[9] P. Podleś and S.L. Woronowicz, On the structure of inhomogeneous quantum groups, hep-th/9412058, UC Berkeley preprint, PAM-631.

[10] S. Zakrzewski, Geometric quantization of Poisson groups - diagonal and soft deformations, Proceedings of the Taniguchi Symposium Symplectic geometry and quantization problems, Sanda (1993), Y. Maeda, H. Omori and A. Weinstein (Eds.), Contemporary Mathematics 179, 1994, 271-285.

[11] S. Zakrzewski, Poisson structures on the Lorentz group, Lett. Math. Phys. 32 (1994), $11-23$.

[12] S. Zakrzewski, Quantum Poincaré group related to $\kappa$-Poincaré algebra, J. Phys. A: Math. Gen. 27 (1994), 2075-2082.

[13] A. Belavin and V.G. Drinfeld, Triangle equations and simple Lie algebras, Sov. Sci. Rev. Math. 4 (1984), 93-165.

[14] S. Zakrzewski, Poisson space-time symmetry and corresponding elementary systems, in: "Quantum Symmetries", Proceedings of the II International Wigner Symposium, Goslar 1991, H.D. Doebner and V.K. Dobrev (Eds.), pp. 111-123.

[15] P. Stachura, in preparation. 
[16] S. Zakrzewski, Poisson homogeneous spaces, in: "Quantum Groups, Formalism and Applications", Proceedings of the XXX Winter School on Theoretical Physics 14-26 February 1994, Karpacz, J. Lukierski, Z. Popowicz, J. Sobczyk (eds.), Polish Scientific Publishers PWN, Warsaw 1995, pp. 629-639 (also hep-th/9412101).

[17] S. Zakrzewski, Phase spaces associated with standard $r$-matrices, q-alg/9511002, Warsaw preprint 1995.

[18] S. Zakrzewski, Poisson Poincaré particle and canonical variables, in: "Generalized Symmetries", Proceedings of the International Symposium on Mathematical Physics, Clausthal, July 27-29, 1993, H.-D. Doebner, V.K. Dobrev and A.G. Ushveridze (Eds.), 1994, pp. 165-171.

[19] S. Zakrzewski, On the classical $\kappa$-particle, in: "Quantum Groups, Formalism and Applications", Proceedings of the XXX Winter School on Theoretical Physics 14-26 February 1994, Karpacz, J. Lukierski, Z. Popowicz, J. Sobczyk (eds.), Polish Scientific Publishers PWN, Warsaw 1995, pp. 573-577 (also: hep-th/9412098).

[20] S. Zakrzewski, Classical mechanical systems based on Poisson symmetry, submitted for the Proceedings of The Second German-Polish Symposium "New Ideas in the Theory of Fundamental Interactions", September 1995, Zakopane, Poland. 